\title{
Generation of human neural retina transcriptome atlas by single cell RNA sequencing
}

Samuel W. Lukowski ${ }^{1,2, \#}$, Camden Y. Lo ${ }^{3, \#}$, Alexei Sharov ${ }^{4}$, Quan H. Nguyen ${ }^{1}$, Lyujie Fang ${ }^{5,6,7}$, Sandy S.C. Hung ${ }^{5,6}$, Ling Zhu ${ }^{8}$, Ting Zhang ${ }^{8}$, Tu Nguyen ${ }^{5,6}$, Anne Senabouth ${ }^{1}$, Jafar S. Jabbari ${ }^{9}$, Emily Welby ${ }^{10}$, Jane C. Sowden ${ }^{10}$, Hayley S. Waugh ${ }^{11}$, Adrienne Mackey ${ }^{11}$, Graeme Pollock ${ }^{11}$, Trevor D. Lamb ${ }^{12}$, Peng-Yuan Wang ${ }^{13,14}$, Alex W. Hewitt ${ }^{5,6,15}$, Mark Gillies ${ }^{8}$, Joseph E. Powell $^{1,16, \wedge}$, Raymond C.B. Wong ${ }^{5,6,17, \wedge}$

${ }^{1}$ Institute for Molecular Bioscience, University of Queensland, Australia

2 The University of Queensland Diamantina Institute, Translational Research Institute, Australia

${ }^{3}$ Private Consultancy

${ }^{4}$ National Institute for Aging, National Institutes of Health, United States

${ }^{5}$ Centre for Eye Research Australia, Australia

${ }^{6}$ Ophthalmology, Department of Surgery, University of Melbourne, Australia

${ }^{7}$ Jinan University, China

${ }^{8}$ Save Sight Institute, University of Sydney, Australia

${ }^{9}$ Australian Genome Research Facility, Australia

10 Stem Cells and Regenerative Medicine Section, NIHR Great Ormond Street Hospital Biomedical Research Centre, UCL Great Ormond Street Institute of Child Health, UK

${ }^{11}$ Lions Eye Donation Services, Australia

12 John Curtin School of Medical Research, The Australian National University, Australia

${ }^{13}$ Department of Chemistry and Biotechnology, Swinburne University of Technology, Australia

14 Center for Human Tissue and organs Degeneration, Institute of Biomedicine and Biotechnology, Shenzhen Institute of Advanced Technology, Chinese Academy of Science, China

${ }^{15}$ Menzies Institute for Medical Research, University of Tasmania, Australia

16 Garvan-Weizmann Centre for Cellular Genomics, Garvan Institute of Medical Research, Australia

${ }^{17}$ Shenzhen Eye Hospital, China

\# equal first-authors.

^equal senior-authors

Lead contact/correspondence: Dr Raymond Wong, Centre for Eye Research Australia, Level 6, 75 Commercial Road, Melbourne, VIC 3004, Australia; Phone: +613 85321962; email: wongcb@unimelb.edu.au 


\section{$\underline{\text { Summary }}$}

The retina is a highly specialized neural tissue that senses light and initiates image processing. Although the functional organisation of specific cells within the retina has been well-studied, the molecular profile of many cell types remains unclear in humans. To comprehensively profile cell types in the human retina, we performed single cell RNA-sequencing on 20,009 cells obtained post-mortem from three donors and compiled a reference transcriptome atlas. Using unsupervised clustering analysis, we identified 18 transcriptionally distinct clusters representing all known retinal cells: rod photoreceptors, cone photoreceptors, Müller glia cells, bipolar cells, amacrine cells, retinal ganglion cells, horizontal cells, retinal astrocytes and microglia. Notably, our data captured molecular profiles for healthy and early degenerating rod photoreceptors, and revealed a novel role of MALAT1 in putative rod degeneration. We also demonstrated the use of this retina transcriptome atlas to benchmark pluripotent stem cell-derived cone photoreceptors and an adult Müller glia cell line. This work provides an important reference with unprecedented insights into the transcriptional landscape of human retinal cells, which is fundamental to our understanding of retinal biology and disease.

\section{Keywords}

Retina, transcriptome, single cell RNA sequencing, photoreceptor subtypes 


\section{Introduction}

The eye is a highly specialised sensory organ in the human body. Sight is initiated by the conversion of light into an electrical signal in the photoreceptors of the neurosensory retina. The rod photoreceptors are responsible for light detection at extremely low luminance, while the cone photoreceptors are responsible for colour detection and operate at moderate and higher levels. Following preprocessing, by horizontal, bipolar and amacrine cells, the resultant signal is transferred via ganglion cells to the brain. Neurotransmitter support is provided by Müller glia, retinal astrocytes and microglial cells. Inherited retinal diseases are becoming the leading cause of blindness in working age adults, with loci in over 200 genes associated with retinal diseases (RetNet: https://sph.uth.edu/retnet/), often involving specific retinal cell types. Knowledge of the transcriptome profile of individual retinal cell types in humans is important to understand the cellular diversity in the retina, as well as the study of retinal genes that contribute to disease in individual retinal cell types. (Hornan et al, 2007a; Mustafi et al, 2016a; Whitmore et al, 2014a; Farkas et al, 2013a; Pinelli et al, 2016a)

The transcriptome profiles of whole human retina from adults (Hornan et al, 2007b; Mustafi et al, 2016b; Whitmore et al, 2014b; Farkas et al, 2013b; Pinelli et al, 2016b) and during fetal development (Hoshino et al, 2017; Kozulin et al, 2009) have been previously described. However, these studies only assayed the averaged transcriptional signatures across all cell types, meaning that the cellular heterogeneity in the retina is lost. As such, the transcriptional pathways that underlie the highly specialised function of many human retinal cell types remain unclear; including the rod and cone photoreceptors, Müller glia cells, horizontal cells, and amacrine cells. Recent advances in RNA sequencing and microfluidic platforms have dramatically improved the accessibility of single cell transcriptomics, with increased throughput at a lower cost. Critically, single-cell microfluidics and low-abundance RNA library chemistries allow accurate profiling of the transcriptome of individual cell types. This has been demonstrated in the mouse, where transcriptome profiles of the mouse retina (Macosko et al, 2015) and retinal bipolar cells (Shekhar et al, 2016) have been described at the single cell level using the Drop-seq method (Macosko et al, 2015). These studies provided a molecular classification of the mouse retina and identified novel markers for specific cell types, as well as novel candidate cell types in the retina. Recently, single cell transcriptomics was used to analyse the human retina. Phillips et al. have profiled a total of 139 adult retina cells using the C1 Fluidigm platform (Phillips et al, 2018), but the limited number of profiled cells presents challenges in the annotation and accurate identification of individual retinal cell types. Moreover, a flow cytometry approach was used to isolate 65 human fetal cone photoreceptors followed by scRNA-seq profiling (Welby et al, 2017).

Herein we report the generation of a human neural retina transcriptome atlas using 20,009 single cells from three donors. Our data provide new insights into the transcriptome profile of major human retinal cell types and establish a high cellular-resolution reference of the human neural 
retina, which will have implications for identification of biomarkers and understanding retinal cell biology and diseases.

\section{$\underline{\text { Results }}$}

Preparation of human neural retinal samples and generation of single cell transcriptome atlas

We obtained post-mortem human adult eyes approved for research purposes following corneal transplantation. As the transcriptome profile of human retina pigment epithelium cells has already been reported (Strunnikova et al, 2010; Liao et al, 2010), we focused solely on the neural retina layers. In this study we extracted the neural retina from twelve donor eyes (Supplementary Table 1). We observed consistent cell viability across retinal tissues retrieved within 15 hours post-mortem (Supplementary figure 1A) and found that donor age does not impact negatively on cell viability in the extracted neural retina (Supplementary figure 1B). To minimize potential risk of mRNA degradation due to reduced cell viability, we selected three donor samples retrieved within 15 hours post-mortem and analysed them with single cell RNA sequencing (scRNA-seq) using the 10X Genomics Chromium platform.

Sequence data from five scRNA-seq libraries derived from the three neural retinal samples were pooled for processing and analysis. From 23,000 cells, we obtained an average of 40,232 reads per cell and 1,665 UMIs (unique transcripts) per cell. Following quality control and filtering in Seurat, our final dataset contained 20,009 cells, which were taken forward for further analysis.

The scRNA-seq data was initially analysed using an unsupervised graph clustering approach implemented in Seurat (version 2.2.1) to classify individual cells into cell subpopulations according to similarities in their transcriptome profiles. Overall, the cells were classified into 18 transcriptionally distinct clusters (Figure 1A, Supplementary figure 2). We first assessed the variation between donor samples and between library preparations (Supplementary table 2, supplementary figure 3 and 4). Interestingly, although many of the identified clusters are present in all three donor retinal samples, we also observed several donor-specific clusters corresponding to rod photoreceptors (Figure 1B, Supplementary figure 3A and 3B). In contrast, we observed minimal variation between two different libraries prepared from the same donor sample, supporting the quality of the scRNA-seq datasets in this study (Supplementary figure 4).

\section{Identification of major cell types in the human retina using scRNA-seq}

Based on known markers (Klimova et al, 2015; Vecino et al, 2016; Shekhar et al, 2016; Macosko et al, 2015; Soto et al, 2008; Imanishi et al, 2002; Blackshaw et al, 2001; Corbo et al, 2007), we were able to assign cell identities to the 16 of the 18 clusters (Figure 1A, 1C, 1D), corresponding to rod photoreceptors (PDE6A, CNGA1, RHO), cone photoreceptors (ARR3, 
GNGT2, GUCA1C), Müller glia (RLBP1/CRALBP), retinal astrocytes (GFAP), microglia (HLADPA1, HLA-DPB1, HLA-DRA), bipolar cells (VSX2, OTX2), retinal ganglion cells (NEFL, GAP43, SNCG), amacrine cells (GAD1, CALB1, CHAT) and horizontal cells (ONECUT1, ONECUT2). The expression of selected marker genes are displayed in $t$-SNE plots (Figure 1D). Two clusters (C5 and C14) express markers from multiple retinal cell types (Supplementary figure 5), thus we were unable to assign cell identities to these 2 clusters and they were excluded from further analysis. Interestingly, our data demonstrated multiple transcriptionally distinct clusters within the rod photoreceptors (6 clusters) and bipolar cells (3 clusters). In contrast, only one cluster was detected for cone photoreceptors, Müller glia, retinal ganglion cells, horizontal cells, amacrine cells, retinal astrocytes and microglia respectively. Correlation analysis confirmed the similarity between clusters within the same cell type (Figure 1E). As expected, we observed high correlations between the expression levels of transcripts within photoreceptor cell types (rod and cones), as well as glial cells (retinal astrocytes and Müller glia) and other retinal neurons (bipolar cells, retinal ganglion cells, amacrine cells and horizontal cells). The composition of cell populations across our three donors show that the majority of the cells in human neural retina were rod photoreceptors $(\sim 74 \%)$ followed by bipolar cells $(\sim 10 \%)$. These results are similar to those reported in mice, where rod photoreceptors and bipolar cells form the majority of cells in the retina (Jeon et al, 1998; Macosko et al, 2015).

To identify genes whose expression was specific to a given cell type, we performed differential gene expression analysis to identify marker genes for each cluster (Figure 1F). We subsequently extracted membrane-related proteins from gene ontology annotations to identify surface markers, which can be used to develop immuno-based methods to isolate primary culture of individual retinal cell types. Supplementary table 3 lists the identified surface markers for individual retinal cell types. We also assessed the gene expression of a panel of commonly known markers in amacrine cells and bipolar cells (Supplementary figure 6-7), as well as a panel of markers for subtype identification recently identified in mouse scRNA-seq studies (Shekhar et al, 2016; Macosko et al, 2015). In summary, we profiled the transcriptomes of all major cell types in the human retina in the presented dataset. Due to their abundance, for the subsequent analyses we focused on the photoreceptors and glial cells.

\section{Profiling healthy and degenerating human rod photoreceptor subpopulations}

We profiled 14,759 rod photoreceptors and showed that they can be classified into six populations with distinct gene expressions (c0, c1, c2, c3, c4, c7; Supplementary table 3). We assessed these six clusters with a panel of 7 known rod or pan-photoreceptor markers (Figure 2A). Our results suggest differential expression patterns among the 7 markers. All 7 rod markers are highly abundant, consistent with previous scRNA-seq studies of mouse and human retina (Phillips et al, 2018; Macosko et al, 2015). The 7 markers showed differential expression patterns in the 6 identified rod photoreceptor clusters. In particular, RHO, GNGT1 and SAG have 
the highest levels of rod marker detected, followed by NRL, ROM1, GNAT1 and CNGA1. We also noted that ROM1 is expressed in both rod and cone photoreceptors, which is consistent with previous studies (Boon et al, 2008). Importantly, many rod photoreceptor clusters consist of a majority of cells from a single donor ( $>90 \%$ for $\mathrm{c} 0, \mathrm{c} 2, \mathrm{c} 4$ and $>80 \%$ for $\mathrm{c} 1, \mathrm{c} 7$; Figure $2 \mathrm{~B}$ ). It is possible that this observation is due to the systematic biases such as differences in tissue retrieval time, age of donors, or other sample preparation variation. The exception is cluster $\mathrm{c} 3$, which is well represented by all three donors.

Next we set out to further define and classify heterogeneity in rod photoreceptors. We observed that MALAT1, a long non-coding RNA that plays a role in retinal homeostasis and disease (Wan et al, 2017), was robustly expressed in $\sim 66 \%$ of the identified rod photoreceptors $(9,750$ cells $)$ while the rest had little to no expression (5,009 cells; Figure 2C). As such, we utilized MALAT1 expression as a discriminator and investigated differences between rod photoreceptors with high expression (MALAT1-hi; > 4.68 normalised transcripts per cell) or low expression (MALAT1-lo; $<4.68$ normalised transcripts per cell). MALAT1-hi and -lo rod photoreceptors were consistently found in each donor and library samples, with MALAT1-hi accounting for $\sim 66 \%, 90 \%$ and $36 \%$ of the rods in donors \#1, \#2 and \#3 respectively (Figure 2D). To further validate this finding, we performed RNA in situ hybridization in another three donor retinal samples. We consistently observed the presence of MALAT1-hi and -lo subpopulations of rod photoreceptors in all retinal samples (Figure 2E, Supplementary figure 8). Together, these results showed the presence of heterogeneity within rod photoreceptors that can be distinguished by MALAT1 expression.

To rule out the possibility that the presence of MALAT1 rod subpopulations is due to donor sample variations, we applied Canonical Correlation Analysis (CCA) to the dataset, via the Seurat package, to correct for technical and batch artefacts. We found that CCA can effectively corrected the donor-specific effect on rod photoreceptor clusters (Figure 3A and 3B). Following CCA correction, we identified 3 rod photoreceptors clusters (CCA0, CCA1, CCA10), which expressed a panel of 7 rod photoreceptor markers and were well represented in all donor samples (Figure 3C). Notably, the majority of cells in CCA0 were low in MALAT1 expression, while CCA1 and CCA10 represented MALAT1-high rod subpopulations (Figure 3D and 3E). This is consistent with our RNA in situ hybridization analysis, where we consistently observed MALAT1-hi and -lo subpopulations of rod photoreceptors in all retinal samples (Supplementary figure 9). Collectively these results supported that the MALAT1 heterogenity in rod photoreceptors are not due to inter-individual variability.

We also considered the possibility that MALAT1-lo rod subpopulations may represent an artefact of 'low quality cells' in scRNA-seq data, due to a low number of sequencing reads or broken cell membrane. In this regard, up-regulated levels of mitochondrial-encoded genes and ribosomal proteins can be used to identify such low quality cells in scRNA-seq data (Ilicic et al, 2016). For our scRNA-seq dataset, we did not observe upregulation in gene expression for a panel of 
ribosomal proteins ( $R P L 41, R P L P 1, R P L 21, R P S 27, R P L 13 A, R P L 36, R P L 39$ and RPS28; Figure $3 \mathrm{~F})$. However, the rod cluster CCA10, representing $1.4 \%$ of rod photoreceptor cells, showed markedly increased levels of mitochondrial-encoded genes (MT-CO2, MT-ND5, MT-ND3, MT$C Y B, M T-N D 1, M T-N D 2, M T-C O 3, M T-A T P 6, M T-C O 1, M T-N D 4)$, suggesting that CCA10 represented a low-quality $M A L A T 1-h i$ rod cluster and was excluded from further analysis.

As we utilised post-mortem retinal samples in this study, we reasoned that MALAT1-lo subpopulation may reflect the early stages of post-mortem degeneration in rod photoreceptors. To determine this, we extracted retinal samples from the same donor at different time points of progressive post-mortem degeneration, with longer time points predicted to have more stressed/dying photoreceptors. Our results showed that there was a high proportion of MALAT1hi rod photoreceptors at 7 hours post-mortem (Figure 4, 95\%). However, we observed a marked decrease in MALAT1 expression in rod photoreceptors at 13 hours post-mortem. Similar results were observed for the three retinal samples processed for scRNA-seq (Supplementary figure 8B). Together, these results demonstrated that MALAT1 is a novel marker for healthy photoreceptors with loss of expression preceding putative cell degeneration. In summary, we showed that scRNA-seq can be used to profile healthy (CCA1) and degenerating rod photoreceptors (CCA0), which can be distinguished by high or low MALAT1 expression levels respectively.

\section{Transcriptome profile of cone subtypes in the human retina}

We detected 564 cone photoreceptor cells in our scRNA-seq data, which are distinguishable from the other cell types by the expression of the cone marker genes ARR3, CNBG3, GNAT2, GNGT2, GRK7, GUCA1C, PDE6C, PDE6H, OPN1LW, RXRG and THRB (Figure 5A). All 11 marker genes analysed show specific expression patterns in the cone cluster (C10). We set out to further assess the composition of the cone cluster. In humans, there are three identified subtypes of cone photoreceptor which can be distinguished by expression of a sole opsin gene: OPN1SWpositive S-cones, OPN1MW-positive M-cones and OPN1LW-positive L-cones respond preferentially to shorter, medium and longer wavelengths responsible for colour vision (Roorda \& Williams, 1999). Notably OPN1LW and OPN1MW exhibit $\sim 98 \%$ sequence homology and are unable to be distinguished by 3' sequencing utilised in this study. By quantifying the number of cells that express the opsin genes, our results showed that the majority of the cone cluster are L/M cones (73.22\%) and S-cones in much lower proportion (3.19\%, Figure 5B), at levels consistent with those estimated by a previous adaptive optics and photo-bleaching study (Roorda \& Williams, 1999). As expected, the identified cone photoreceptors only express either OPN1SW or $O P N 1 L W / M W$ (Figure 3C).

To further study the molecular differences and identify molecular markers for the cone subtypes, we performed differential gene expression analysis to determine genes that can distinguish the cone subtypes. Our results identified a panel of genes that differentially marked S-cones (e.g. 
CCDC136, RAMP1, LY75, CADM3, TFPI2, CRHBP, RAB17, UPB1, RRAD, SLC12A1) and L/M-cones (e.g. THRB, KIF2A, LBH, PGP, CHRNA3, AHI1, LIMA1; Figure 3D). Together these results detailed the molecular profiles and identified marker genes that can distinguish the cone subtypes in human.

\section{Assessment of glial cells in human retina}

Next, we focused on two related glial cell types in the human retina, the Müller glia and the retinal astrocytes. Our scRNA-seq data has profiled a total of 2,723 Müller glia cells which classified into a single cluster (C9) and 49 retinal astrocytes which form a single cluster (C16). Figure 5E shows the expression of a panel of 9 commonly used markers for Müller glia and retinal astrocytes. Our results demonstrated that many of these markers are present in both Müller glia and retinal astrocytes at differential expression levels. VIM, GLUL and S100A1 marked both Müller glia and retinal astrocytes at high expression levels. GFAP represents a reliable marker for retinal astrocytes, and its expression is consistent with a previous report (Vecino et al, 2016). Notably, Müller glia are low in GFAP expression, indicating they are not in an activated state commonly caused by stresses and reactive gliosis (Fernández-Sánchez et al, 2015). The $S 100 B$ is also expressed in retinal astrocytes at varying levels but absent in Müller glia. Conversely, Müller glia can be distinguished from retinal astrocytes by high expression levels of $R L B P 1$, and $R G R$ to a lesser extent. Together these results provide insights into the differential expression patterns of known glial markers in Müller glia and retinal astrocytes in human.

As glial cell proliferation has been linked to a range of pathological conditions including retinal gliosis and retinal injury (Subirada et al, 2018), this provides a means of assessing the health of the profiled retinas. We assigned a cell cycle phase score to each cell using gene expression signatures for the G1, S, G2, and G2/M phases (Kowalczyk et al, 2015); Supplementary figure 9). We determined that most of the Müller glial cells expressed genes indicative of cells in G1 phase $(75 \%)$, suggesting they are not proliferative. These results demonstrated the absence of hallmarks of gliosis/retinal injury and support the quality of the donor retinas profiled.

\section{Using the human neural retina transcriptome atlas for benchmarking}

To demonstrate the use of our dataset as a benchmarking reference, we compared the scRNA-seq profiles of distinct cell types generated using alternative methods, including fetal human cone photoreceptors, human induced pluripotent stem cell derived-cone photoreceptors (hiPSC-cone; (Welby et al, 2017), and a sample of adult human retina with 139 cells (Phillips et al, 2018). Correlation analysis demonstrated that the adult human retina sample showed highest similarity to rod photoreceptor (0.63, Supplementary figure 10), which is expected as rod photoreceptors represent the majority of the cells in the retina. Interestingly, our results also showed that the 
transcriptome of hiPSC-cone after 15 weeks of differentiation exhibited the highest similarity to cone photoreceptors, and low similarities to all other retinal cell types (Figure 6A, Supplementary figure 10). In particular, hiPSC-cone showed high similarities to fetal cone photoreceptors and adult cone photoreceptors ( 0.71 and 0.61 respectively), and a much lower similarity to adult rod photoreceptors (0.33). In support of this, principal component analysis demonstrated that the hiPSC-cone are closer to fetal cone photoreceptors, rather than the adult counterpart (Figure 6B, Supplementary figure 10). These results confirmed direct differentiation of hiPSCs to cone photoreceptors with good quality, and the hiPSC-derived cone photoreceptors are closer to fetal origin compared to their adult counterpart.

In another benchmarking example, we set out to assess the potential differences between in vitro cell lines compared to adult cells in vivo. In this regard, we compared the spontaneously immortalised human Müller glia cell line MIO-M1 (Lawrence et al, 2007; Limb et al, 2002) to all the retinal cell types identified in our dataset. Using scRNA-seq, we profiled 7,150 MIO-M1 cells with 23,987 reads per cell post-normalization corresponding to 3,421 detected genes. Unsupervised clustering and t-SNE analysis showed that the MIO-M1 cells formed one cluster that is transcriptionally distinct from all retina cell types identified in the human neural retina dataset (Fig 6C, Supplementary figure 10). Correlation analysis showed that MIO-M1 displayed similarities to retinal glial cells, with higher similarity to astrocytes compared to Müller glia (0.63 and 0.46 respectively, Fig 6D). In particular, we identified that MIO-M1 express high levels of thymosin beta 4 gene (TMSB4X), which has been linked to glioma malignancy (Wirsching et al, 2013), as well as the calcyclin gene (S100A6), which is implicated in macular or cone associated diseases (Yoshida et al, 2004); Figure 4E). Together, our results highlighted the similarities and differences of MIO-M1 to adult retinal glial cells in human.

\section{$\underline{\text { Discussion }}$}

The data presented here describe the generation of a detailed reference transcriptome atlas of the human neural retina at the single-cell level. The establishment of reference transcriptome maps for individual cell types in the retina provide unprecedented insights into the signals that define retinal cell identity and advance our understanding of the retina. This human neural retina transcriptome data can be used as a benchmark to assess the quality and maturity of pluripotent stem cell-derived retinal cells, such as retinal ganglion cells (Gill et al, 2016; Kobayashi et al, 2018; Sluch et al, 2015) and photoreceptors (Lakowski et al, 2018). We also confirmed that a relatively low level of sequencing depth ( 40,232 reads per cell) is sufficient for identification and classification of major cell types in a complex tissue like retina. However, we acknowledge that increasing the sequencing depth and cell number could improve the ability to distinguish retinal subtypes that are highly complex, such as the amacrine cells and the retinal ganglion cells. 
One of the most interesting observations is the presence of heterogeneous subpopulations within known retinal cell types. This highlights the sensitivity of using a scRNA-seq approach to capture and classify retinal cell types in an unbiased manner. In particular, our results demonstrated the presence of two rod photoreceptor subpopulations in post-mortem retina that display differential expression of MALAT1. Notably, the presence of MALAT1-hi and -lo rod subpopulations were consistently observed in all post-mortem samples analysed $(\mathrm{n}=7)$. We further showed that MALAT1-lo subpopulations represent early degenerating rod photoreceptors, a finding that has not previously been reported in human or any other species. Previous studies have demonstrated a role of MALAT1 in regulating the survival of retinal ganglion cells (Li et al, 2017) and in pathogenesis of retinal pigment epithelium cells (Yang et al, 2016). However, the functional role of MALAT1 in photoreceptors remained unclear. Our results demonstrated that loss of MALAT1 expression in rod photoreceptors following putative post-mortem degeneration, and suggests MALAT1 as a potential target to enhance rod photoreceptor survival and retinal preservation. Future studies are warranted to investigate the functional role of MALAT1 in photoreceptors. Our transcriptome data also revealed rod photoreceptor clusters specific to particular donor retinas and we showed that application of the CCA method could effectively correct for these donor/batch variations in rod photoreceptors. Further studies with a larger number of donor samples will allow testing of the feasibility of using scRNA-seq to comprehensively analyse the retina in different individuals, such as assessment of the effects of aging or degenerative retinal diseases.

Another outcome of this study is the assessment of biomarkers that allow identification of major retinal cell types and subtypes. Our results provide new insights into the cone photoreceptor subtypes in human. The cone subtypes are traditionally categorized based on expression of different opsins that allowed for cellular detection of light at various wavelength. While the Scones are structurally different from the other two cone subtypes, the L-cones and M-cones are structurally similar and difficult to distinguish from each other, except for the opsin they expressed (Viets et al, 2016). We report the first description of the transcriptome profiles of Scones in adult human and highlight novel marker genes that can be used to distinguish them. We also identified the transcriptome and novel marker genes for L/M-cones, however given the high sequence homology, particularly at the 3' end, of OPN1MW and OPN1LW, we could not confidently separate L-cones and M-cones. In addition, we show that many of the known Müller glia markers are often expressed in retinal astrocytes, and we also provide a detailed assessment of commonly used retinal glial markers showing the differential expression pattern between Müller glia and retinal astrocytes.

Finally, our results highlighted the use of this neural retina transcriptome atlas to benchmark retinal cells derived from stem cells or primary cultures. A major goal of pluripotent stem cell research is to derive cells that are similar to those in adults in vivo, which is important for development of stem cell disease models and regenerative medicine (Hung et al, 2017). Our 
analysis shows that hiPSC-derived cone photoreceptors are highly similar to both fetal and adult cones in comparison with all other major retinal cell types. We show that hiPSC-derived cells are more fetal-like than adult-like, which is consistent with other studies (Handel et al, 2016; Baxter et al, 2015). We also benchmark a commonly used Müller glia cell line MIO-M1. Our results showed that while this cell line exhibits similarities to adult retinal glial cells, there are also some differences between MIO-M1 and adult Müller glia, such as high expression of the gliomarelated gene thymosin beta4 (TMSB4X) in MIO-M1. Previous reports have also described differences in gene expression in MIO-M1 to Müller glia, and showed that MIO-M1 express markers for post-mitotic retinal neurons and neural stem cells (Lawrence et al, 2007; Hollborn et $a l, 2011)$. These results highlighted the potential effects of prolonged in vitro culture of primary retinal cells. Collectively, we showed that the human neural retina transcriptome atlas provides an important benchmarking resource to assess the quality of derived retinal cells, which would have implications for stem cell and neuroscience research.

One of the limitations of this study is the finite number of profiled cell types less frequently represented in the retina such as the amacrine cells and the retinal ganglion cells, which are known to be highly complex. The presented dataset is limited in power to accurately identify differences in the transcriptomes of the subtypes in amacrine and retinal ganglion cells. With the identification of surface markers for these cell types in this study, this work lays the foundation for future research using selection and enrichment (Shekhar et al, 2016) of these and other cell types (such as horizontal cells or retinal microglia) to improve the resolution of the human neural retina transcriptome atlas. Another limitation is the use of 3' gene expression profiling, which presents a challenge for distinguishing L-cones and M-cones. Given the high sequence homology of $O P N 1 L W$ and $O P N 1 M W(98 \%)$, future studies using full-length mRNA sequencing of single cone photoreceptor cells would provide greater distinction and classification accuracy of the $O P N 1 M W$ and $O P N 1 L W$-positive cells.

This study describes the transcriptome of human neural retina at a single cell level and is the first to identify the transcriptome of all major human retinal cell types. Our findings shed light on the molecular differences between subpopulations within the rod photoreceptors and the cone photoreceptors. The presented dataset provides an important roadmap to define the genetic signals in major cell types in the human retina and can be used as a benchmark to assess the quality of stem cell-derived cells or primary retinal cells.

\section{$\underline{\text { Online Methods }}$}

\section{Human retina collection}

Collection of patient samples was approved by the Human Research Ethics committee of the Royal Victorian Eye and Ear Hospital (HREC13/1151H) and Save Sight Institute (16/282) and carried out in accordance with the approved guidelines. For scRNA-seq, post-mortem eye globes 
were collected by the Lions Eye Donation Service (Royal Victorian Eye and Ear Hospital) for donor cornea transplantation. The remaining eye globes were used for dissection to extract the neural retina. The lens, iris and vitreous were removed and the choroid/RPE layers were excluded from the sample collection. Following extraction, the neural retinal samples were dissociated and processed for scRNA-seq right away. Neural retina samples were dissociated into single cells in dissociation solution (2mg/ml papain, 120 Units/ml DNase I) for 15 minutes. The dissociated neural retina was filtered to ensure single cell suspension using a 30 $\mu \mathrm{m}$ MACS Smart Strainer (Miltenyi). For sample from Patient SC, the Dead Cell Removal kit (Miltenyi) was utilised to remove dead cells prior to scRNA-seq. However, in our hands we found that the Dead Cell Removal kit only had a modest improvement in the cell viability ( $\sim 8 \%$ improvement, data not shown).

Single cell RNA sequencing (scRNA-seq)

Single cells from three independent neural retina samples were captured in five batches using the 10X Chromium system (10X Genomics). The cells were partitioned into gel bead $\square$ in $\square$ emulsions and barcoded cDNA libraries, then prepared using the single cell 3' mRNA kit (V2; 10X Genomics). Single cell libraries were sequenced in $100 \mathrm{bp}$ paired-end configuration using an Illumina Hi-Seq 2500 at the Australian Genome Research Facility.

\section{Bioinformatics processing}

The 10X Genomics cellranger pipeline (version 2.1.0; (Zheng et al, 2017) was used to generate fastq files from raw Illumina BCL files (mkfastq) and aligned to the human genome reference GRCh38 using the included STAR alignment software (Dobin et al, 2013). Next, cellranger count was used to generate read count matrices. To overcome the stringent threshold implemented in cellranger that discards real cells under certain conditions, such as populations of cells with a low RNA content, the --force-cells parameter was set to 3000 for the donor 1 library and 5000 for donor 2 and 3 libraries. Using the barcode rank plot, these parameters were selected to increase the number of detected cells for further analysis. The cellranger aggregation function (aggr) was used to combine the 5 libraries and normalize the between-sample library size differences. The full scRNA-seq dataset is available in ArrayExpress under accession number EMTAB-7316.

Data were imported into the Seurat single cell analysis software (v2.0.1; https://github.com/satijalab/seurat). Quality control of sequenced libraries was performed to remove outlier cells and genes. Cells with 200-2500 detected genes and expressing < 10\% mitochondrial genes were retained. Genes were retained in the data if they were expressed in $\geq 3$ cells. Additional cell-cell normalization was performed using the LogNormalize method, and inherent variation caused by mitochondrial gene expression and the number of unique molecular identifiers (UMIs) per cell was regressed out. 
Clustering was performed on PCA-reduced expression data using the top 20 principal components using the graph-based shared nearest neighbour method (SNN) which calculates the neighborhood overlap (Jaccard index) between every cell and its nearest neighbors.

Prediction of the cell cycle phase of individual retinal cells was performed in Seurat using cell cycle-specific expression data (Kowalczyk et al, 2015). Briefly, genetic markers associated with G2/M and S phase were used to assign cell scores, and cells expressing neither of the G2/M or S phase markers were classified as being in G1 phase.

Sequencing data for fetal (scRNA-seq) and hiPSC-derived cone photoreceptors (bulk RNA-seq) was obtained from ArrayExpress using the accession numbers E-MTAB-6057 and E-MTAB6058 (Welby et al, 2017). Gene expression matrices were generated from the fastq files using the STAR aligner software. scRNA-seq data from 72 cells were quality-controlled, filtered and then normalised with the scran algorithm (Lun et al, 2016) as described (Welby et al, 2017), using the ascend (https://github.com/IMB-Computational-Genomics-Lab/ascend) package in R, which resulted in 63 high quality single cell transcriptomes. Bulk RNA-seq data generated from 6 hiPSC-derived cone photoreceptor cultures was filtered such that each gene was represented by at least 10 counts in all samples and normalisation was performed in edgeR using the trimmed mean of M method (Robinson \& Oshlack, 2010). Pre-processed scRNA-seq data generated from adult retina (Phillips et al, 2018) was obtained from the Gene Expression Omnibus (GSE98556).

\section{Canonical correlation analysis}

Canonical correlation analysis (CCA) was applied to correct donor-specific effects observed in the rod photoreceptor populations. This was achieved by separating the raw data into 5 samplespecific datasets, which were then used as inputs for the RunMultiCCA function in Seurat. For the CCA analysis, we used the most highly variable genes that were shared by all 5 samples and the recombined data was aligned using the first $20 \mathrm{CC}$ dimensions, selected by biweight midcorrelation (bicor) analysis. Aligned cells were reclustered in Seurat using the first 20 aligned $\mathrm{CC}$ dimensions at a resolution of 0.6

\section{Identification of retinal cell types}

Cell types were classified using differential expression analysis, which compared each cluster to all others combined using the Wilcoxon method in Seurat to identify cluster-specific marker genes. Each retained marker gene was expressed in a minimum of $25 \%$ of cells and at a minimum $\log _{2}$ fold change threshold of 0.25 .

In paired cluster analyses, differentially expressed genes were considered significant if the adjusted p-value was less than 0.01 (Benjamini-Hochberg correction for multiple testing) and the absolute $\log _{2}$ expression fold change was $\geq 0.5$. 


\section{Mapping cells between subpopulations in different samples}

To compare subpopulations identified in the merged dataset (5 samples), we applied scGPS (single cell Global Projection between Subpopulations), a machine learning procedure to select optimal gene predictors and to build prediction models that can estimate between-subpopulation transition scores. The transition scores are the probability of cells from one subpopulation that are in the same class with the cells in the other subpopulation. The scores, therefore, estimate the similarity between any two subpopulations. Here, we compared three main subpopulations from sample Retina 2A with all subpopulations in the sample Retina 2B. The source code of the scGPS method is available with open-access (https://github.com/IMB-Computational-GenomicsLab/scGPS).

\section{Correlation of scRNA-seq data with retinal cell types}

The mean expression levels of cells in each cluster were calculated and used to calculate Pearson's correlations in a pairwise manner with each of the other clusters and results were deemed significant if the correlation P-value was less than 0.01 .

\section{Pathway analysis}

Gene enrichment analysis was performed using Enrichr (Kuleshov et al, 2016). The combined score, computed by taking the log of p-value from the Fisher exact test and multiplying by the $\mathrm{z}$ score of the deviation of the expected rank, was used to determine the enrichment ranking for pathways, othologies, transcription factor network and protein network analysis.

\section{Fluorescent in situ hybridization}

Donor retinas were first dissected from the eye cup. The retinal tissues were subjected to $30 \%$ sucrose cryoprotection and were then frozen in $-80^{\circ} \mathrm{C}$. Sections were cut on a cryostat (Leica CM3050S) and mounted on glass slides (SuperFrostPlus). The retinal samples were fixed in $3.7 \%$ (vol/vol) formaldehyde and hybridized with Stellaris RNA FISH probes (Biosearch Technologies) against MALAT1 labeled with Quasar 570, following the manufacturer's instructions. Briefly, samples were incubated with Quasar 570-labeled probes at $125 \mathrm{nM}$ in hybridization buffer and hybridized 5 hours at $37^{\circ} \mathrm{C}$, followed by nuclear counterstain using DAPI. The samples are imaged using a ZEISS confocal laser-scanning microscope (ZEISS, LSM700).

\section{Author Contributions}

HW, AM coordinated and collected the human donor retinas, GP provided the funding for human donor tissue collection, LF, TN, JJ, SH, RW, LZ, TZ conducted the experiments; SL, CL, AS, RW, QN, EW, JS, TL, LZ, TZ processed and/or analysed the data; PYW, AH, JP, MG and RW contributed to experimental design and data analysis; SL, AH, JP and RW wrote the manuscript. 


\section{Acknowledgements}

The human Müller cell line Moorfields/Institute of Ophthalmology- Müller 1 (MIO-M1) was obtained from the UCL Institute of Ophthalmology, London, UK. The authors want to thank David Mackey and Holly Chinnery for critical discussion and helpful feedback for this study, and Prema Finn for technical assistance for the collection of human donor samples. This work was supported by funding from the Ophthalmic Research Institute of Australia (RW, SL), the University of Melbourne De Brettville Trust (RW) and the Kel and Rosie Day Foundation (RW). JCS is supported by GOSHCC. The Centre for Eye Research Australia receives operational infrastructure support from the Victorian Government.

\section{Competing Interests statement}

The authors have no conflict of interest to declare. 


\section{$\underline{\text { References }}$}

Baxter M, Withey S, Harrison S, Segeritz C-P, Zhang F, Atkinson-Dell R, Rowe C, Gerrard DT, Sison-Young R, Jenkins R, Henry J, Berry AA, Mohamet L, Best M, Fenwick SW, Malik H, Kitteringham NR, Goldring CE, Piper Hanley K, Vallier L, et al (2015) Phenotypic and functional analyses show stem cell-derived hepatocyte-like cells better mimic fetal rather than adult hepatocytes. J. Hepatol. 62: 581-589

Blackshaw S, Fraioli RE, Furukawa T \& Cepko CL (2001) Comprehensive analysis of photoreceptor gene expression and the identification of candidate retinal disease genes. Cell 107: 579-589

Boon CJF, den Hollander AI, Hoyng CB, Cremers FPM, Klevering BJ \& Keunen JEE (2008) The spectrum of retinal dystrophies caused by mutations in the peripherin/RDS gene. Prog. Retin. Eye Res. 27: 213-235

Corbo JC, Myers CA, Lawrence KA, Jadhav AP \& Cepko CL (2007) A typology of photoreceptor gene expression patterns in the mouse. Proc. Natl. Acad. Sci. U. S. A. 104: 12069-12074

Dobin A, Davis CA, Schlesinger F, Drenkow J, Zaleski C, Jha S, Batut P, Chaisson M \& Gingeras TR (2013) STAR: ultrafast universal RNA-seq aligner. Bioinformatics 29: 15-21

Farkas MH, Grant GR, White JA, Sousa ME, Consugar MB \& Pierce EA (2013a) Transcriptome analyses of the human retina identify unprecedented transcript diversity and $3.5 \mathrm{Mb}$ of novel transcribed sequence via significant alternative splicing and novel genes. BMC Genomics 14: 486

Farkas MH, Grant GR, White JA, Sousa ME, Consugar MB \& Pierce EA (2013b) Transcriptome analyses of the human retina identify unprecedented transcript diversity and $3.5 \mathrm{Mb}$ of novel transcribed sequence via significant alternative splicing and novel genes. BMC Genomics 14: 486

Fernández-Sánchez L, Lax P, Campello L, Pinilla I \& Cuenca N (2015) Astrocytes and Müller Cell Alterations During Retinal Degeneration in a Transgenic Rat Model of Retinitis Pigmentosa. Front. Cell. Neurosci. 9: 484

Gill KP, Hung SSC, Sharov A, Lo CY, Needham K, Lidgerwood GE, Jackson S, Crombie DE, Nayagam BA, Cook AL, Hewitt AW, Pébay A \& Wong RCB (2016) Enriched retinal ganglion cells derived from human embryonic stem cells. Sci. Rep. 6: 30552

Handel AE, Chintawar S, Lalic T, Whiteley E, Vowles J, Giustacchini A, Argoud K, Sopp P, Nakanishi M, Bowden R, Cowley S, Newey S, Akerman C, Ponting CP \& Cader MZ (2016) Assessing similarity to primary tissue and cortical layer identity in induced pluripotent stem cell-derived cortical neurons through single-cell transcriptomics. Hum. Mol. Genet. 25: 989-1000

Hollborn M, Ulbricht E, Rillich K, Dukic-Stefanovic S, Wurm A, Wagner L, Reichenbach A, 
Wiedemann P, Limb GA, Bringmann A \& Kohen L (2011) The human Müller cell line MIO-M1 expresses opsins. Mol. Vis. 17: 2738-2750

Hornan DM, Peirson SN, Hardcastle AJ, Molday RS, Cheetham ME \& Webster AR (2007a) Novel retinal and cone photoreceptor transcripts revealed by human macular expression profiling. Invest. Ophthalmol. Vis. Sci. 48: 5388-5396

Hornan DM, Peirson SN, Hardcastle AJ, Molday RS, Cheetham ME \& Webster AR (2007b) Novel retinal and cone photoreceptor transcripts revealed by human macular expression profiling. Invest. Ophthalmol. Vis. Sci. 48: 5388-5396

Hoshino A, Ratnapriya R, Brooks MJ, Chaitankar V, Wilken MS, Zhang C, Starostik MR, Gieser L, La Torre A, Nishio M, Bates O, Walton A, Bermingham-McDonogh O, Glass IA, Wong ROL, Swaroop A \& Reh TA (2017) Molecular Anatomy of the Developing Human Retina. Dev. Cell 43: 763-779.e4

Hung SSC, Khan S, Lo CY, Hewitt AW \& Wong RCB (2017) Drug discovery using induced pluripotent stem cell models of neurodegenerative and ocular diseases. Pharmacol. Ther. 177: $32-43$

Ilicic T, Kim JK, Kolodziejczyk AA, Bagger FO, McCarthy DJ, Marioni JC \& Teichmann SA (2016) Classification of low quality cells from single-cell RNA-seq data. Genome Biol. 17: 29

Imanishi Y, Li N, Sokal I, Sowa ME, Lichtarge O, Wensel TG, Saperstein DA, Baehr W \& Palczewski K (2002) Characterization of retinal guanylate cyclase-activating protein 3 (GCAP3) from zebrafish to man. Eur. J. Neurosci. 15: 63-78

Jeon CJ, Strettoi E \& Masland RH (1998) The major cell populations of the mouse retina. J. Neurosci. 18: 8936-8946

Kim J-W, Yang H-J, Oel AP, Brooks MJ, Jia L, Plachetzki DC, Li W, Allison WT \& Swaroop A (2016) Recruitment of Rod Photoreceptors from Short-Wavelength-Sensitive Cones during the Evolution of Nocturnal Vision in Mammals. Dev. Cell 37: 520-532

Klimova L, Antosova B, Kuzelova A, Strnad H \& Kozmik Z (2015) Onecut1 and Onecut2 transcription factors operate downstream of Pax6 to regulate horizontal cell development. Dev. Biol. 402: 48-60

Kobayashi W, Onishi A, Tu H-Y, Takihara Y, Matsumura M, Tsujimoto K, Inatani M, Nakazawa T \& Takahashi M (2018) Culture Systems of Dissociated Mouse and Human Pluripotent Stem Cell-Derived Retinal Ganglion Cells Purified by Two-Step Immunopanning. Invest. Ophthalmol. Vis. Sci. 59: 776-787

Kowalczyk MS, Tirosh I, Heckl D, Rao TN, Dixit A, Haas BJ, Schneider RK, Wagers AJ, Ebert BL \& Regev A (2015) Single-cell RNA-seq reveals changes in cell cycle and differentiation programs upon aging of hematopoietic stem cells. Genome Res. 25: 1860-1872 
Kozulin P, Natoli R, O’Brien KMB, Madigan MC \& Provis JM (2009) Differential expression of anti-angiogenic factors and guidance genes in the developing macula. Mol. Vis. 15: 45-59

Kuleshov MV, Jones MR, Rouillard AD, Fernandez NF, Duan Q, Wang Z, Koplev S, Jenkins SL, Jagodnik KM, Lachmann A, McDermott MG, Monteiro CD, Gundersen GW \& Ma'ayan A (2016) Enrichr: a comprehensive gene set enrichment analysis web server 2016 update. Nucleic Acids Res. 44: W90-7

Lakowski J, Welby E, Budinger D, Di Marco F, Di Foggia V, Bainbridge JWB, Wallace K, Gamm DM, Ali RR \& Sowden JC (2018) Isolation of Human Photoreceptor Precursors via a Cell Surface Marker Panel from Stem Cell-Derived Retinal Organoids and Fetal Retinae. Stem Cells Available at: http://dx.doi.org/10.1002/stem.2775

Lawrence JM, Singhal S, Bhatia B, Keegan DJ, Reh TA, Luthert PJ, Khaw PT \& Limb GA (2007) MIO-M1 cells and similar muller glial cell lines derived from adult human retina exhibit neural stem cell characteristics. Stem Cells 25: 2033-2043

Liao J-L, Yu J, Huang K, Hu J, Diemer T, Ma Z, Dvash T, Yang X-J, Travis GH, Williams DS, Bok D \& Fan G (2010) Molecular signature of primary retinal pigment epithelium and stem-cell-derived RPE cells. Hum. Mol. Genet. 19: 4229-4238

Li H-B, You Q-S, Xu L-X, Sun L-X, Abdul Majid AS, Xia X-B \& Ji D (2017) Long NonCoding RNA-MALAT1 Mediates Retinal Ganglion Cell Apoptosis Through the PI3K/Akt Signaling Pathway in Rats with Glaucoma. Cell. Physiol. Biochem. 43: 2117-2132

Limb GA, Salt TE, Munro PMG, Moss SE \& Khaw PT (2002) In vitro characterization of a spontaneously immortalized human Müller cell line (MIO-M1). Invest. Ophthalmol. Vis. Sci. 43: 864-869

Lun ATL, Bach K \& Marioni JC (2016) Pooling across cells to normalize single-cell RNA sequencing data with many zero counts. Genome Biol. 17: 75

Macosko EZ, Basu A, Satija R, Nemesh J, Shekhar K, Goldman M, Tirosh I, Bialas AR, Kamitaki N, Martersteck EM, Trombetta JJ, Weitz DA, Sanes JR, Shalek AK, Regev A \& McCarroll SA (2015) Highly Parallel Genome-wide Expression Profiling of Individual Cells Using Nanoliter Droplets. Cell 161: 1202-1214

Mustafi D, Kevany BM, Bai X, Golczak M, Adams MD, Wynshaw-Boris A \& Palczewski K (2016a) Transcriptome analysis reveals rod/cone photoreceptor specific signatures across mammalian retinas. Hum. Mol. Genet. 25: 4376-4388

Mustafi D, Kevany BM, Bai X, Golczak M, Adams MD, Wynshaw-Boris A \& Palczewski K (2016b) Transcriptome analysis reveals rod/cone photoreceptor specific signatures across mammalian retinas. Hum. Mol. Genet. 25: 4376-4388

Oh ECT, Cheng H, Hao H, Jia L, Khan NW \& Swaroop A (2008) Rod differentiation factor NRL activates the expression of nuclear receptor NR2E3 to suppress the development of cone photoreceptors. Brain Res. 1236: 16-29 
Phillips MJ, Jiang P, Howden S, Barney P, Min J, York NW, Chu L-F, Capowski EE, Cash A, Jain S, Barlow K, Tabassum T, Stewart R, Pattnaik BR, Thomson JA \& Gamm DM (2018) A Novel Approach to Single Cell RNA-Sequence Analysis Facilitates In Silico Gene Reporting of Human Pluripotent Stem Cell-Derived Retinal Cell Types. Stem Cells 36: 313-324

Pinelli M, Carissimo A, Cutillo L, Lai C-H, Mutarelli M, Moretti MN, Singh MV, Karali M, Carrella D, Pizzo M, Russo F, Ferrari S, Ponzin D, Angelini C, Banfi S \& di Bernardo D (2016a) An atlas of gene expression and gene co-regulation in the human retina. Nucleic Acids Res. 44: 5773-5784

Pinelli M, Carissimo A, Cutillo L, Lai C-H, Mutarelli M, Moretti MN, Singh MV, Karali M, Carrella D, Pizzo M, Russo F, Ferrari S, Ponzin D, Angelini C, Banfi S \& di Bernardo D (2016b) An atlas of gene expression and gene co-regulation in the human retina. Nucleic Acids Res. 44: 5773-5784

Robinson MD \& Oshlack A (2010) A scaling normalization method for differential expression analysis of RNA-seq data. Genome Biol. 11: R25

Roorda A \& Williams DR (1999) The arrangement of the three cone classes in the living human eye. Nature 397: 520-522

Shekhar K, Lapan SW, Whitney IE, Tran NM, Macosko EZ, Kowalczyk M, Adiconis X, Levin JZ, Nemesh J, Goldman M, McCarroll SA, Cepko CL, Regev A \& Sanes JR (2016) Comprehensive Classification of Retinal Bipolar Neurons by Single-Cell Transcriptomics. Cell 166: 1308-1323.e30

Sluch VM, Davis C-HO, Ranganathan V, Kerr JM, Krick K, Martin R, Berlinicke CA, MarshArmstrong N, Diamond JS, Mao H-Q \& Zack DJ (2015) Differentiation of human ESCs to retinal ganglion cells using a CRISPR engineered reporter cell line. Sci. Rep. 5: 16595

Soto I, Oglesby E, Buckingham BP, Son JL, Roberson EDO, Steele MR, Inman DM, Vetter ML, Horner PJ \& Marsh-Armstrong N (2008) Retinal Ganglion Cells Downregulate Gene Expression and Lose Their Axons within the Optic Nerve Head in a Mouse Glaucoma Model. Journal of Neuroscience 28: 548-561

Strunnikova NV, Maminishkis A, Barb JJ, Wang F, Zhi C, Sergeev Y, Chen W, Edwards AO, Stambolian D, Abecasis G, Swaroop A, Munson PJ \& Miller SS (2010) Transcriptome analysis and molecular signature of human retinal pigment epithelium. Hum. Mol. Genet. 19: $2468-2486$

Subirada PV, Paz MC, Ridano ME, Lorenc VE, Vaglienti MV, Barcelona PF, Luna JD \& Sánchez MC (2018) A journey into the retina: Müller glia commanding survival and death. Eur. J. Neurosci. 47: 1429-1443

Tsukamoto Y, Morigiwa K, Ueda M \& Sterling P (2001) Microcircuits for night vision in mouse retina. J. Neurosci. 21: 8616-8623 
Vecino E, Rodriguez FD, Ruzafa N, Pereiro X \& Sharma SC (2016) Glia-neuron interactions in the mammalian retina. Prog. Retin. Eye Res. 51: 1-40

Viets K, Eldred K \& Johnston RJ Jr (2016) Mechanisms of Photoreceptor Patterning in Vertebrates and Invertebrates. Trends Genet. 32: 638-659

Wan P, Su W \& Zhuo Y (2017) Precise long non-coding RNA modulation in visual maintenance and impairment. J. Med. Genet. 54: 450-459

Welby E, Lakowski J, Di Foggia V, Budinger D, Gonzalez-Cordero A, Lun ATL, Epstein M, Patel A, Cuevas E, Kruczek K, Naeem A, Minneci F, Hubank M, Jones DT, Marioni JC, Ali RR \& Sowden JC (2017) Isolation and Comparative Transcriptome Analysis of Human Fetal and iPSC-Derived Cone Photoreceptor Cells. Stem Cell Reports 9: 1898-1915

Whitmore SS, Wagner AH, DeLuca AP, Drack AV, Stone EM, Tucker BA, Zeng S, Braun TA, Mullins RF \& Scheetz TE (2014a) Transcriptomic analysis across nasal, temporal, and macular regions of human neural retina and RPE/choroid by RNA-Seq. Exp. Eye Res. 129: 93-106

Whitmore SS, Wagner AH, DeLuca AP, Drack AV, Stone EM, Tucker BA, Zeng S, Braun TA, Mullins RF \& Scheetz TE (2014b) Transcriptomic analysis across nasal, temporal, and macular regions of human neural retina and RPE/choroid by RNA-Seq. Exp. Eye Res. 129: 93-106

Wirsching H-G, Krishnan S, Florea A-M, Frei K, Krayenbühl N, Hasenbach K, Reifenberger G, Weller M \& Tabatabai G (2013) Thymosin beta 4 gene silencing decreases stemness and invasiveness in glioblastoma. Brain 137: 433-448

Yang S, Yao H, Li M, Li H \& Wang F (2016) Long Non-Coding RNA MALAT1 Mediates Transforming Growth Factor Beta1-Induced Epithelial-Mesenchymal Transition of Retinal Pigment Epithelial Cells. PLoS One 11: e0152687

Yoshida S, Mears AJ, Friedman JS, Carter T, He S, Oh E, Jing Y, Farjo R, Fleury G, Barlow C, Hero AO \& Swaroop A (2004) Expression profiling of the developing and mature Nrl -/mouse retina: identification of retinal disease candidates and transcriptional regulatory targets of Nrl. Hum. Mol. Genet. 13: 1487-1503

Zheng GXY, Terry JM, Belgrader P, Ryvkin P, Bent ZW, Wilson R, Ziraldo SB, Wheeler TD, McDermott GP, Zhu J, Gregory MT, Shuga J, Montesclaros L, Underwood JG, Masquelier DA, Nishimura SY, Schnall-Levin M, Wyatt PW, Hindson CM, Bharadwaj R, et al (2017) Massively parallel digital transcriptional profiling of single cells. Nat. Commun. 8: 14049 


\section{Figure Legends}

Figure 1: Single cell transcriptome atlas for human neural retina. t-SNE visualization of 20,009 human retinal cells colored by A) annotation of 18 transcriptionally distinct clusters (C0$\mathrm{C} 17$ ) and B) their distribution in 3 donor retina samples. C) Feature expression heatmap showing expression patterns of major retinal class markers across 16 retinal cell clusters. The size of each circle depicts the percentage of cells expressing the marker within the cluster. Brown colour indicates $\geq 10$ nTrans (number of transcripts). D) t-SNE plots showing expression of a set of selected marker genes for major retinal classes. E) Correlation matrix for the identified 18 clusters. The upper triangle depicts the z-value for correlation and the lower triangle depicts the correlation coefficient for gene expression in clusters. F) Heatmap of differentially expressed genes used to classify cell types for each cluster compared to all other clusters for the 18 retinal cell clusters. The rows correspond to top 10 genes most selectively upregulated in individual clusters ( $\mathrm{p}<0.01$, Benjamini-Hochberg correction) and the columns show individual cells ordered by cluster (C0-C17). 
bioRxiv preprint doi: https://doi.org/10.1101/425223; this version posted August 3, 2019. The copyright holder for this preprint (which was not certified by peer review) is the author/funder, who has granted bioRxiv a license to display the preprint in perpetuity. It is made available under aCC-BY-NC 4.0 International license.

A

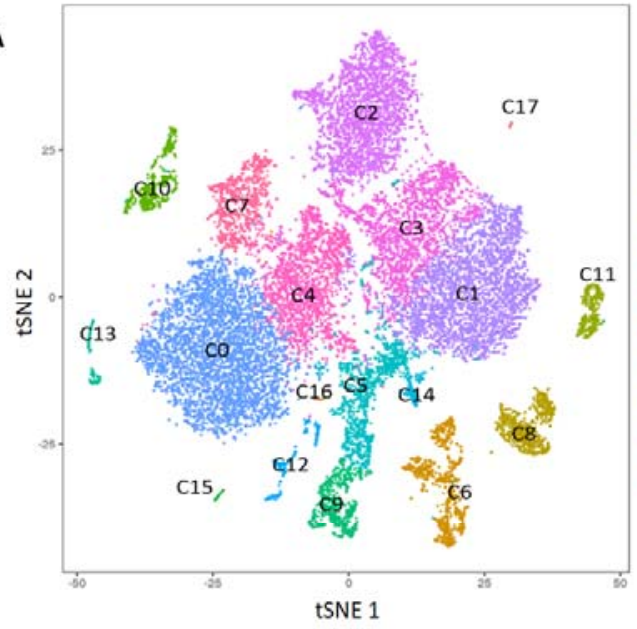

C

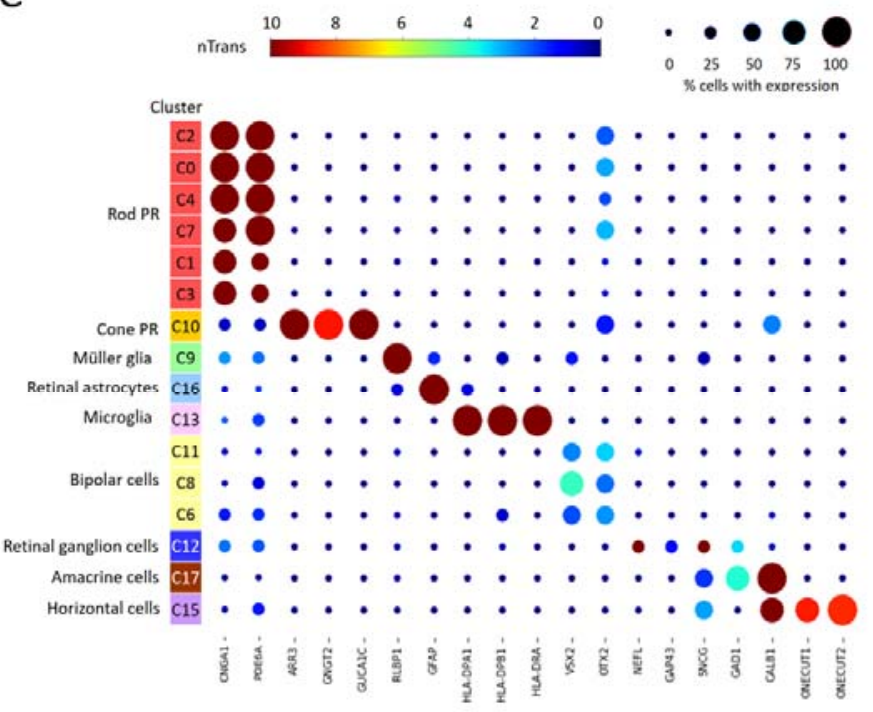

B

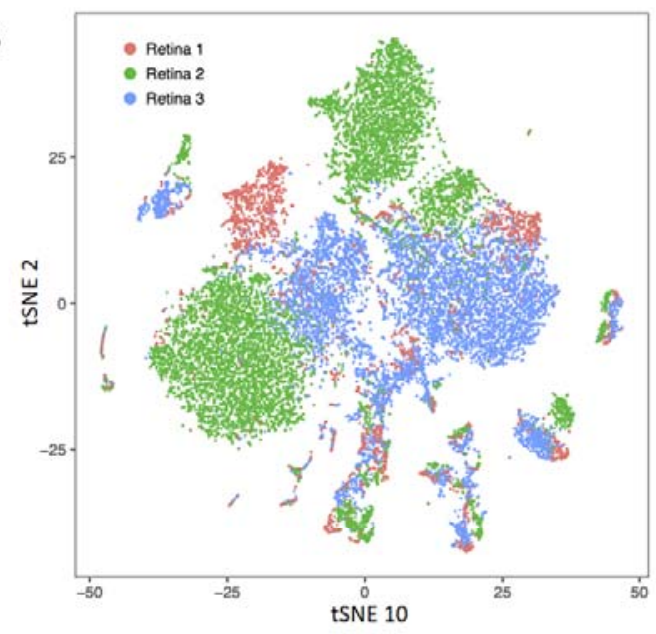

D
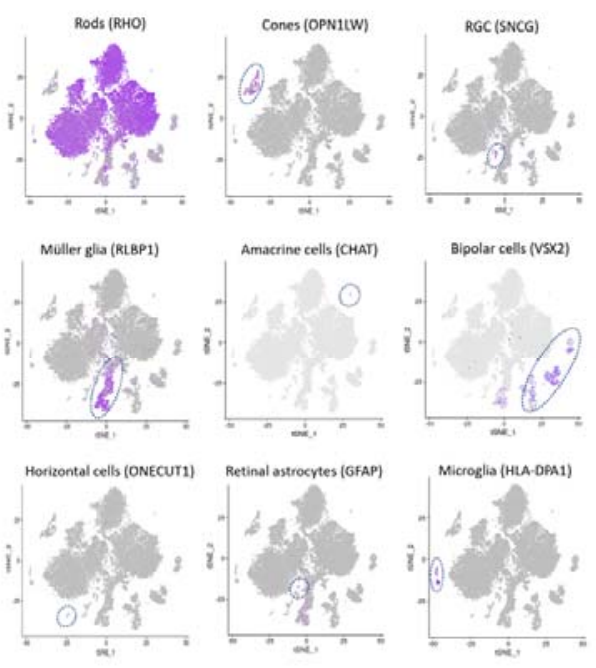

E

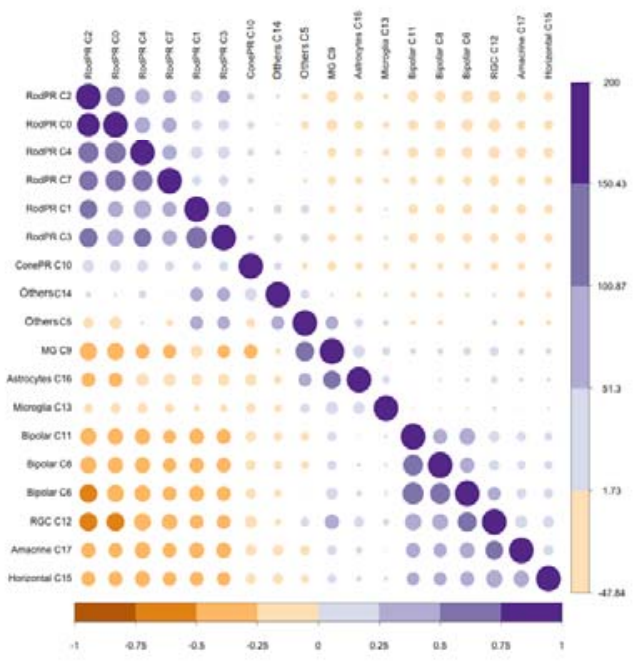

$\mathrm{F}$

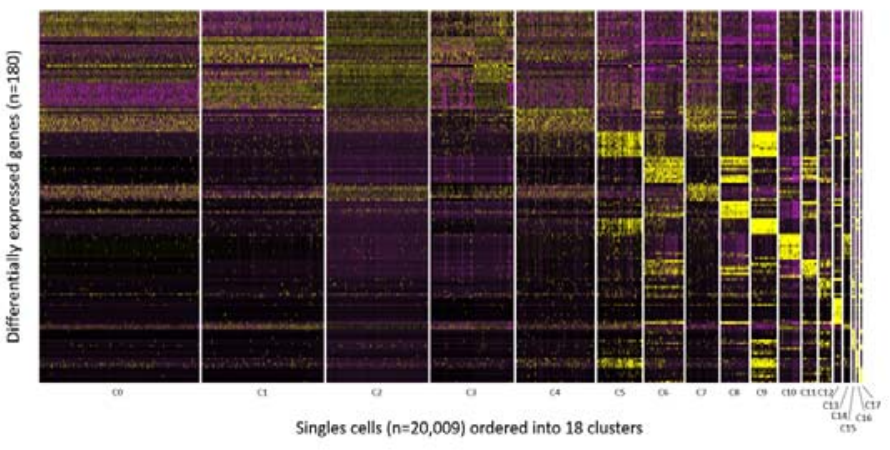


Figure 2: Identification of MALAT1-hi and MALAT1-lo subpopulations of rod photoreceptors. A) Feature expression heatmap of a panel of known marker genes for rod photoreceptors across the identified 16 retinal cell clusters. Brown colour indicates $\geq 100 \mathrm{nTrans}$ (number of transcripts). B) Representation of the three donor retina samples in the six rod photoreceptor clusters. C) Violin plot showing high or low expression levels of MALATI in rod photoreceptor clusters. D) Distribution of rod photoreceptor populations with high MALAT1 expression (MALAT1-hi) or low MALAT1 expression (MALAT1-lo) in three donor retina samples. E) Fluorescent in situ hybridization analysis of human peripheral retina showing heterogeneous levels of MALAT1 expression in the rod photoreceptors located in the outer nuclear layer (ONL). INL: inner nuclear layer; OPL: outer plexiform layer. Scale bar $=20 \mu \mathrm{m}$.

A

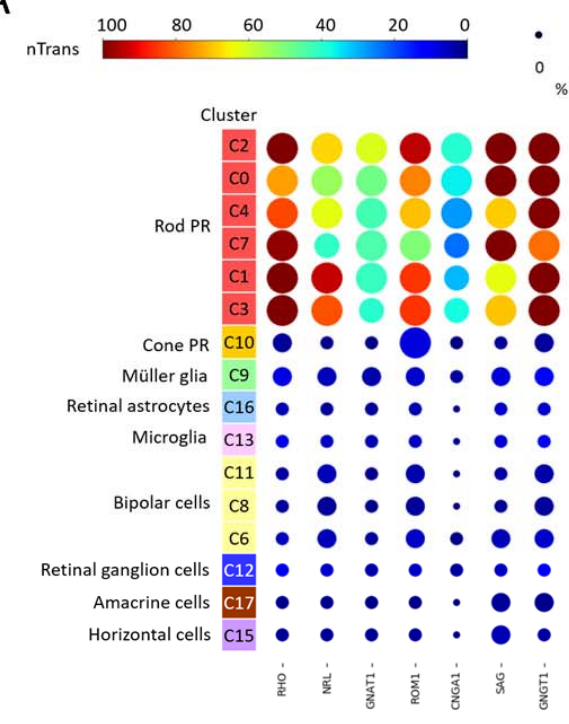

B

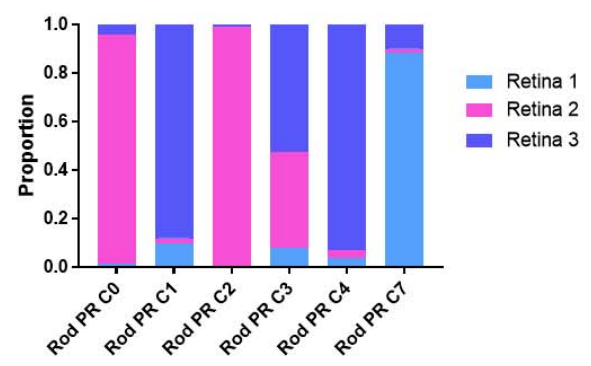

C

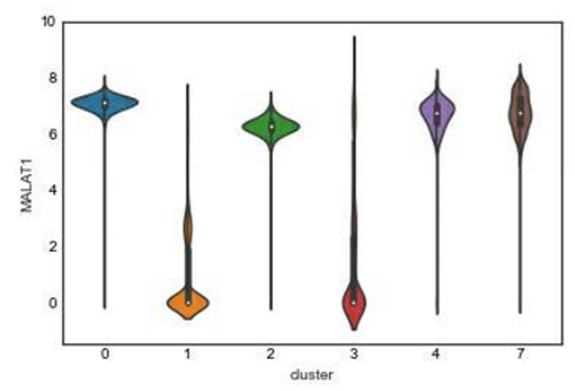

D

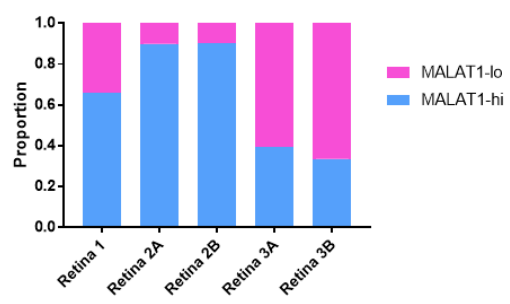

E

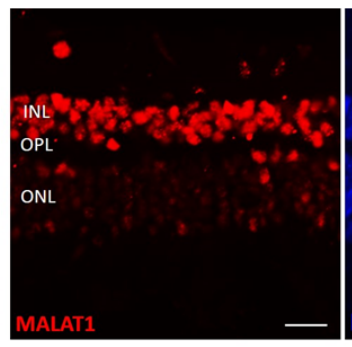

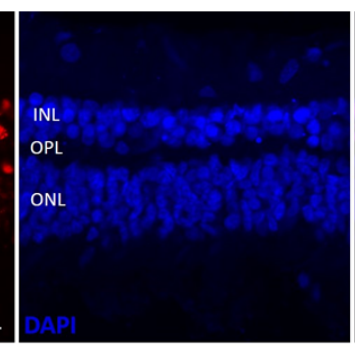

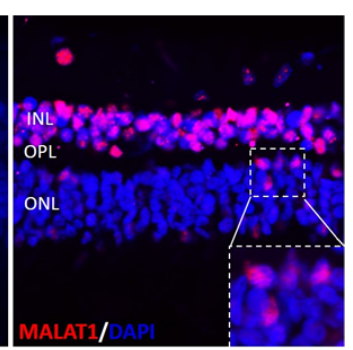


Figure 3: MALAT1 subpopulations of rod photoreceptors is not due to donor variation. Canonical correlation analysis was used to effectively correct donor-specific variations in rod photoreceptors. A) t-SNE visualization of human retinal cells colored by annotation of 13 transcriptionally distinct clusters (CCA0-CCA12) and B) their distribution in 3 donor retina samples. C) Feature expression heatmap showing expression patterns of 7 rod photoreceptor markers across 12 retinal cell clusters. The size of each circle depicts the percentage of cells expressing the marker within the cluster. Brown colour indicates $\geq 50$ nTrans (number of transcripts). D) t-SNE plots showing expression of MALAT1. E) Expression pattern of MALAT1 in the rod photoreceptor showing MALAT1-hi (CCA1, CCA10) and MALAT1-lo (CCA0) subpopulations. x-axis depicts normalized transcript levels. F) t-SNE plots showing expression of major ribosomal genes. G) Heatmap of differentially expressed genes between the two MALAT1-hi clusters CCA1 and CCA10. The rows correspond to top 10 genes most selectively upregulated in individual clusters ( $\mathrm{p}<0.01$, Benjamini-Hochberg correction) and the columns show individual cells ordered in CCA1 and CCA10. 
bioRxiv preprint doi: https://doi.org/10.1101/425223; this version posted August 3, 2019. The copyright holder for this preprint (which was not certified by peer review) is the author/funder, who has granted bioRxiv a license to display the preprint in perpetuity. It is made available under aCC-BY-NC 4.0 International license.

A

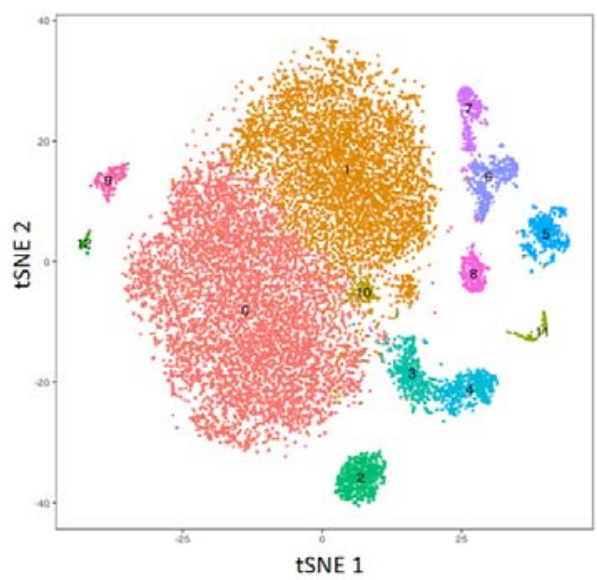

C
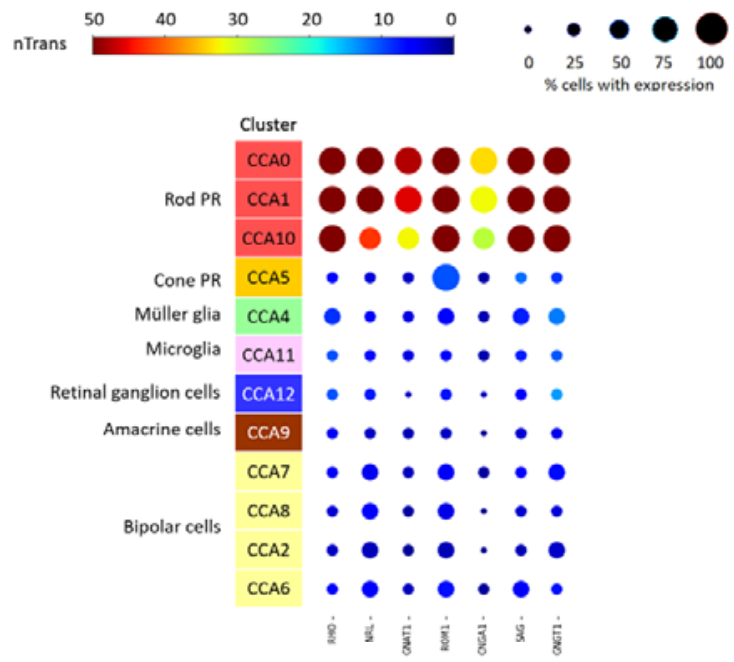

$\mathrm{F}$
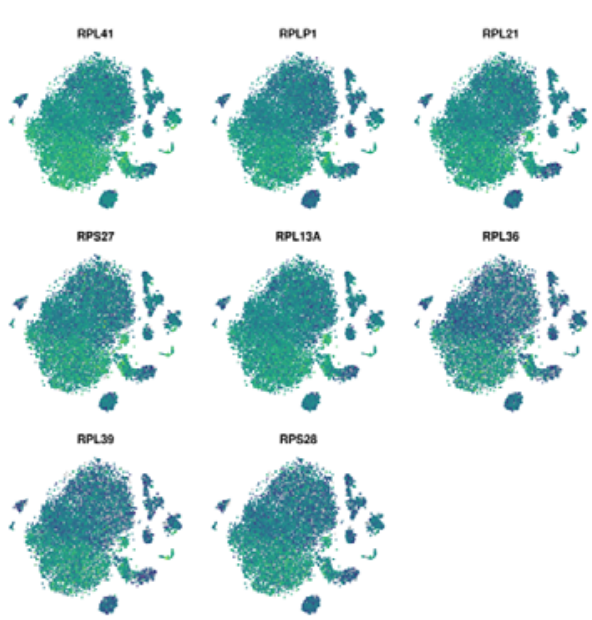

B

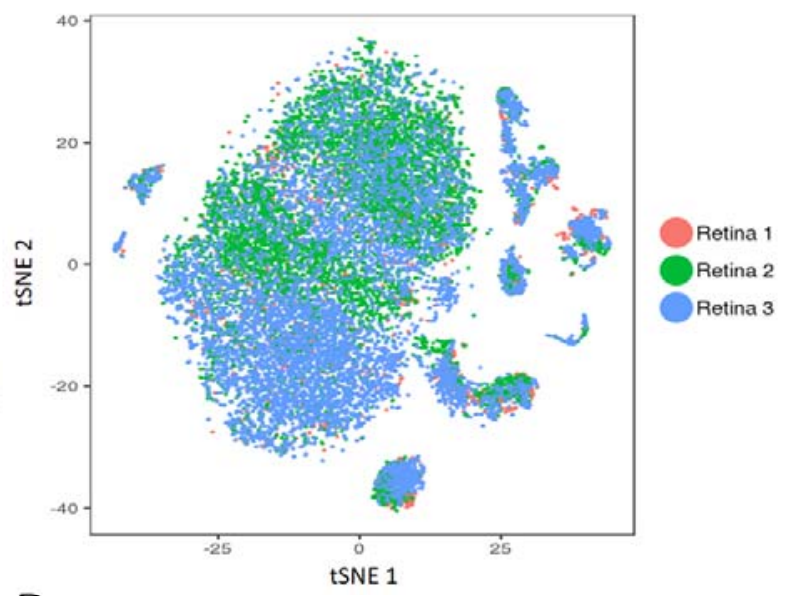

D

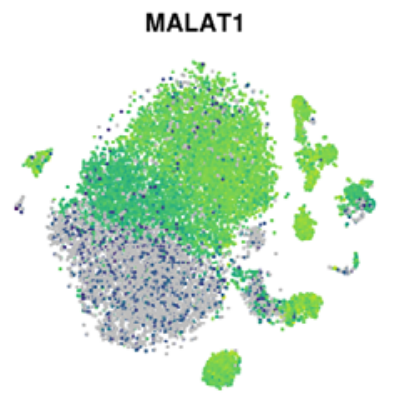

$\mathrm{E}$

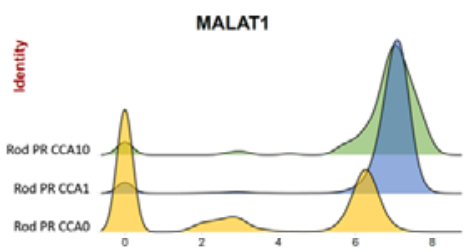

G

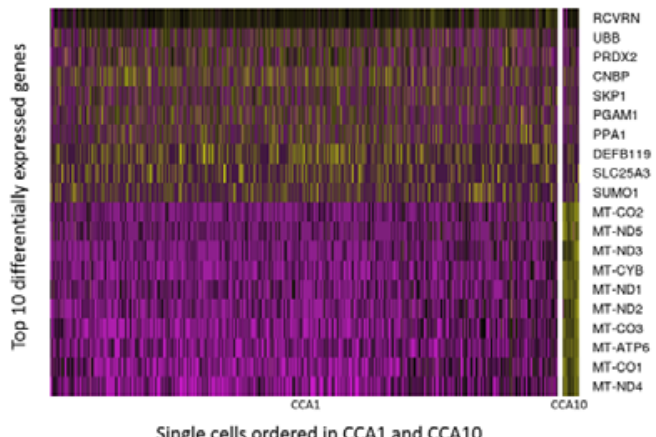


bioRxiv preprint doi: https://doi.org/10.1101/425223; this version posted August 3, 2019. The copyright holder for this preprint (which was not certified by peer review) is the author/funder, who has granted bioRxiv a license to display the preprint in perpetuity. It is made available under aCC-BY-NC 4.0 International license.

Figure 4: Loss of MALAT1 expression in rod photoreceptors following post-mortem degeneration. Fluorescent in situ hybridization analysis of the same donor human peripheral retina at different time points post-mortem (7 and 13 hours, Retina 7), showing decreases in MALAT1-hi rod subpopulations in the outer nuclear layer (ONL) at later time point. INL: inner nuclear layer; OPL: outer plexiform layer. Scale bar $=20 \mu \mathrm{m}$. White arrows indicated MALAT1hi rod photoreceptors.

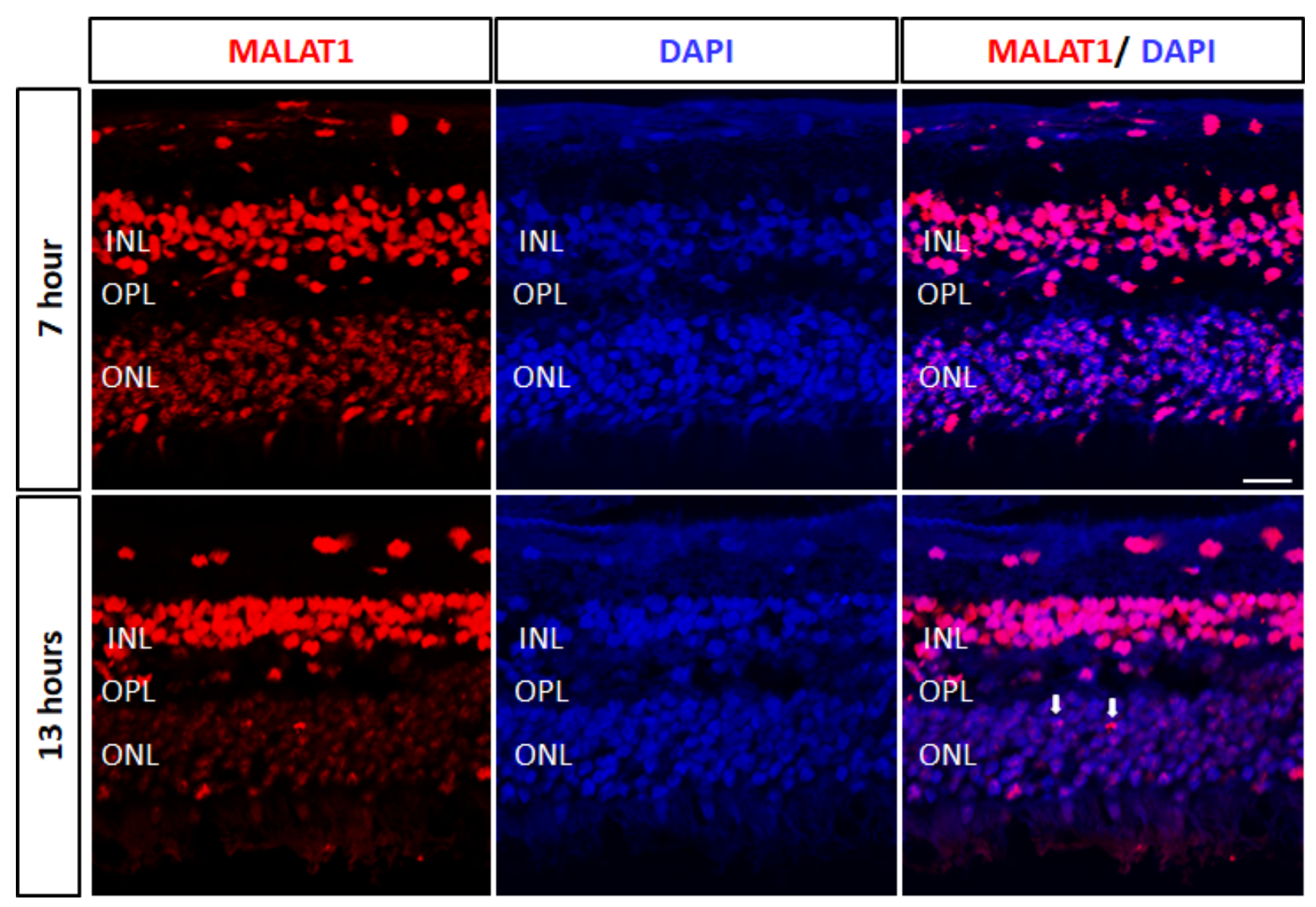


Figure 5: Assessment of cone photoreceptor and glial cell types in human retina . A) Feature expression heatmap showing the expression of 11 known cone photoreceptor markers across 16 retinal cell clusters. Brown colour indicates $\geq 10$ nTrans (number of transcripts). B) The proportion of cone photoreceptor subtypes identified in $\mathrm{C} 10$ cluster, based on expression of OPN1LW / OPN1MW (L/M cones) and OPN1SW (S cones). C) Scatter plots showing expression of $O P N 1 L W / O P N 1 M W$ or OPN1SW in individual cone photoreceptors for C10 cluster. The colour depicts expression level for $O P N 1 L W / O P N 1 M W$ in individual cells. D) Heatmap of top 20 differentially expressed genes between L/M cones and $\mathrm{S}$ cones. The colour depicts normalised gene expression (z-score capped at 2.5). E) Expression pattern of glial markers in Muller glia (C9) and retinal astrocytes (C16). X-axis depicts normalized transcript levels. 
A

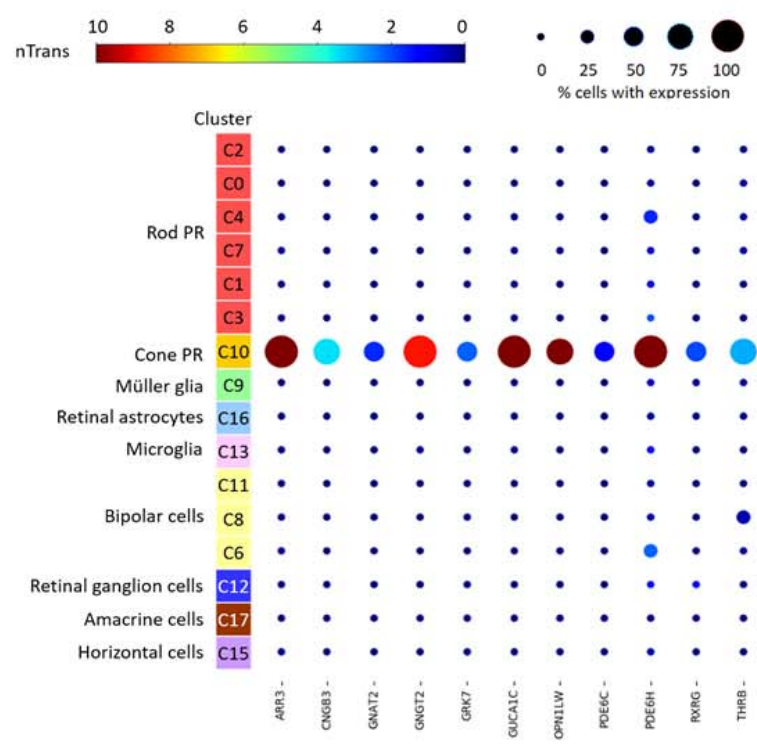

B
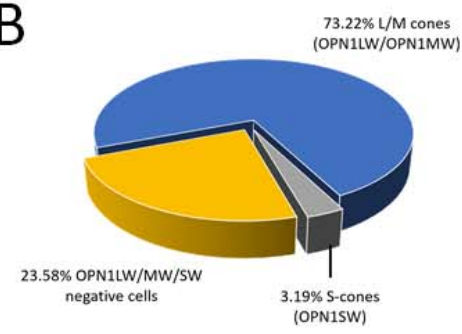

C

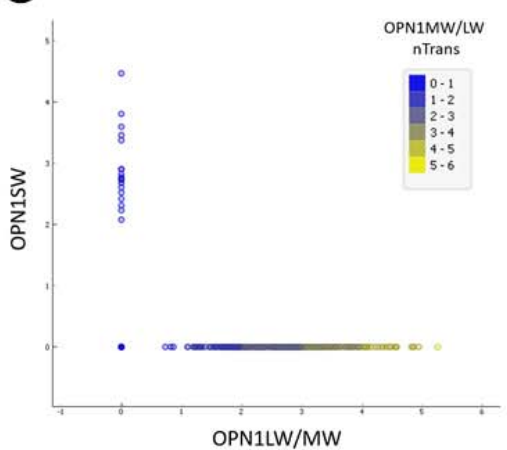

D

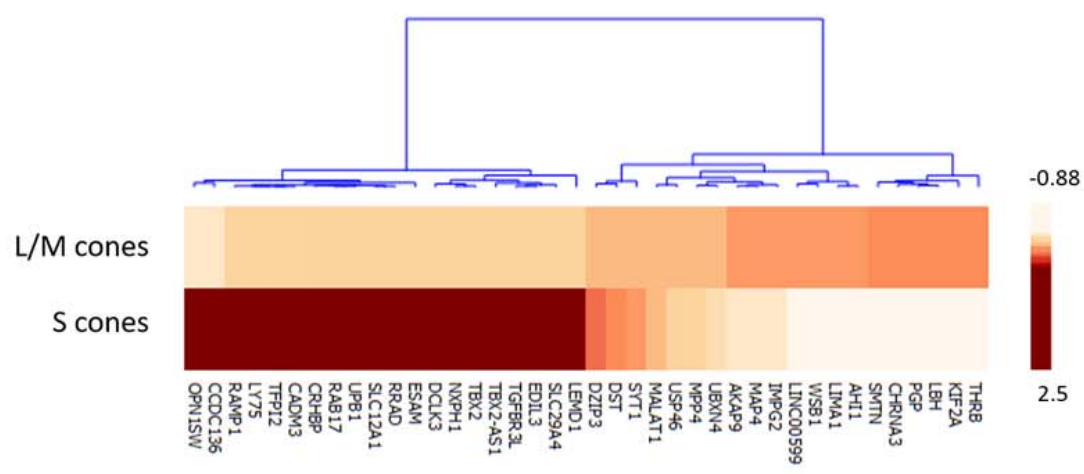

$\mathrm{E}$
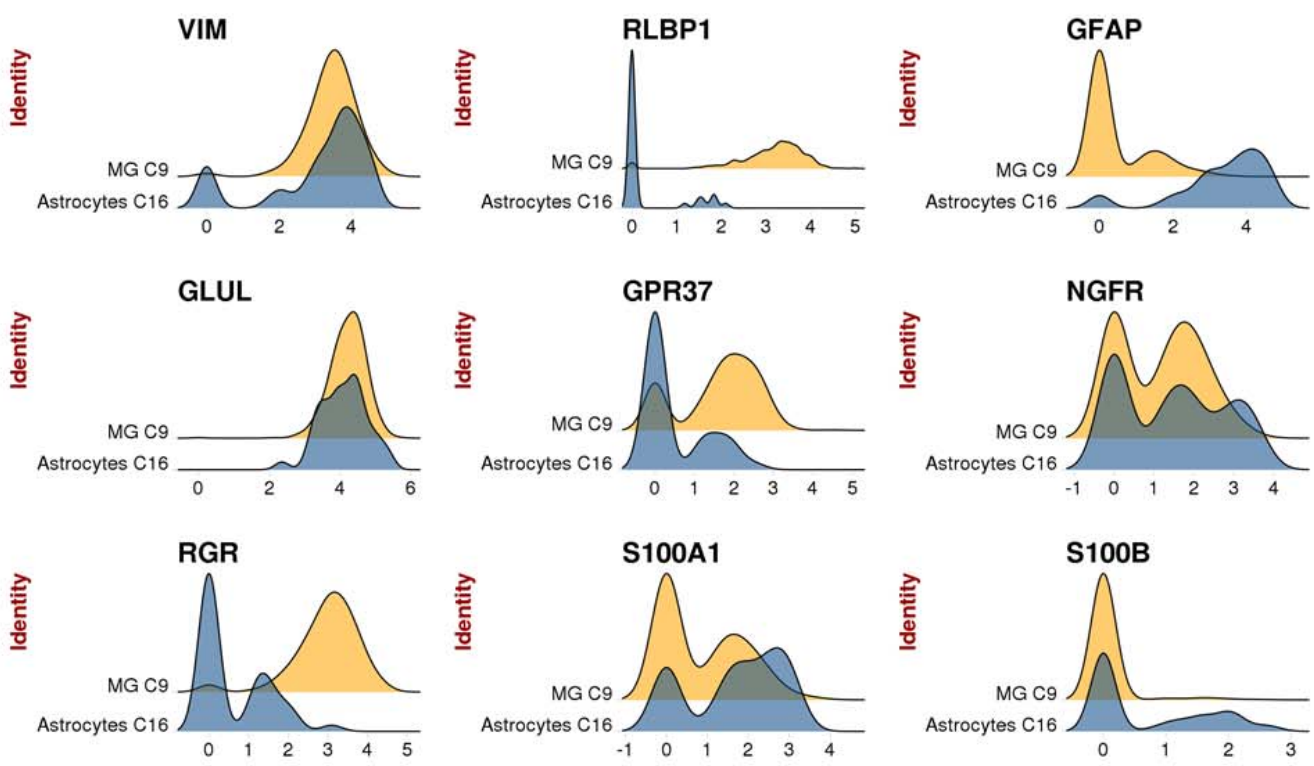

Normalised expression level 
Figure 6: Benchmarking retinal cells using the human neural retina atlas. A) Correlation analysis of scRNA-seq data of hiPSC-derived cone photoreceptors (week 15) against fetal cone photoreceptors (Welby et al, 2017), as well as adult cone and rod photoreceptors from this human neural retina atlas. B) Principal component analysis to assess transcriptome similarity of hiPSC-derived cone photoreceptors to fetal and adult photoreceptors. C) t-SNE analysis of the human Müller glia cell line MIO-M1 with the retinal cell types identified in this human neural retina atlas. D) Correlation analysis of MIO-M1 with all major human retinal cell types. E) Top ranked differentially expressed genes identified in MIO-M1 compared to other retinal cell types based on logistic regression score. 
bioRxiv preprint doi: https://doi. org/10.1101/425223; this version posted August 3, 2019. The copyright holder for this preprint (which was not certified by peer review) is the author/funder, who has granted bioRxiv a license to display the preprint in perpetuity. It is made available under aCC-BY-NC 4.0 International license.
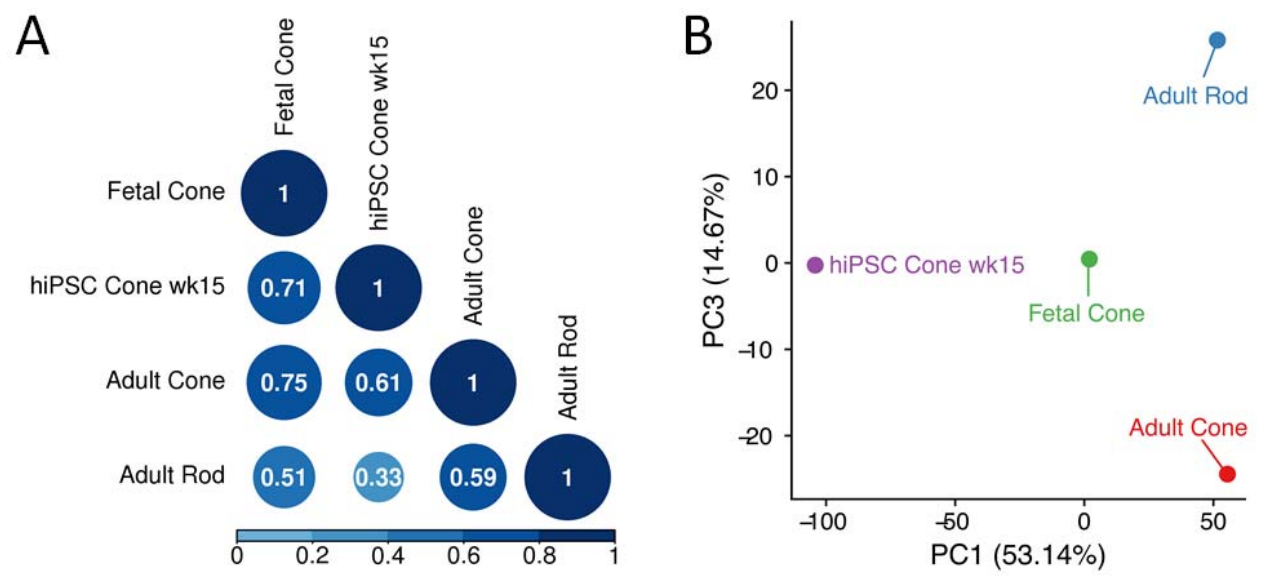

C
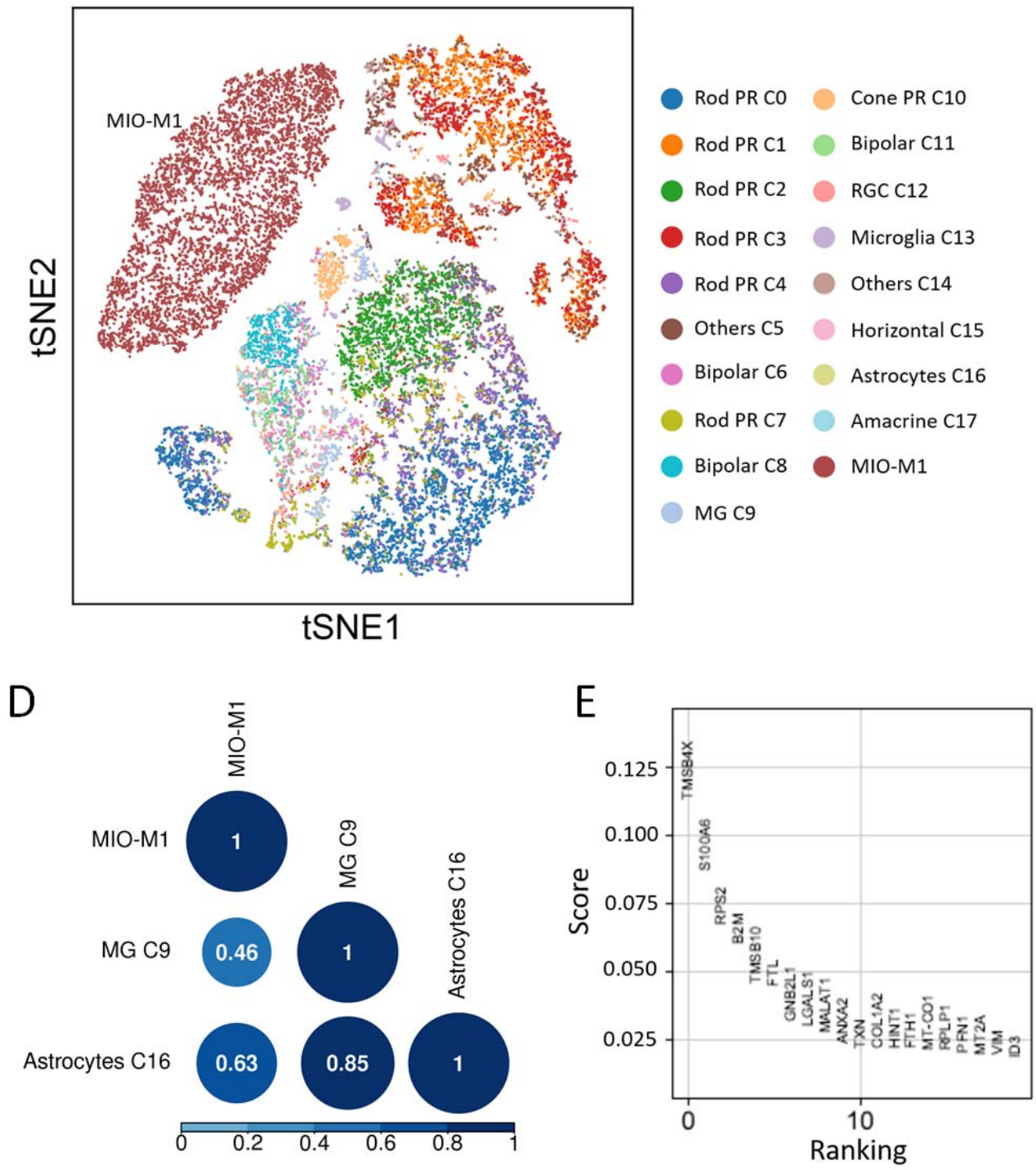


\section{Supplementary figures and tables:}

Supplementary table 1: Details for donor retina samples.

\begin{tabular}{|c|c|c|c|c|c|c|c|c|c|c|}
\hline Retina & $\begin{array}{l}\text { Patient } \\
\text { ID }\end{array}$ & $\begin{array}{l}\text { Eye } \\
\text { bulb }\end{array}$ & Sex & $\begin{array}{c}\text { Age } \\
\text { (years) }\end{array}$ & $\begin{array}{l}\text { Retrieval } \\
\text { time (hrs) }\end{array}$ & $\begin{array}{c}\text { Ocular } \\
\text { complications }\end{array}$ & Assay & $\begin{array}{l}\text { scRNA-seq } \\
\text { Library }\end{array}$ & $\begin{array}{c}\text { Targeted } \\
\text { cell } \\
\text { number }\end{array}$ & $\begin{array}{l}\text { Captured } \\
\text { cell number } \\
\text { after QC }\end{array}$ \\
\hline 1 & $17-010$ & Right & $\mathrm{F}$ & 80 & 11.5 & $\begin{array}{c}\text { Cataract on left } \\
\text { eye }\end{array}$ & $\begin{array}{l}\text { scRNAseq, } \\
\text { cell viability }\end{array}$ & A & 4000 & 2122 \\
\hline \multirow[t]{2}{*}{2} & $\mathrm{SC}$ & Left & M & 42 & 6.2 & - & $\begin{array}{l}\text { scRNAseq, } \\
\text { cell viability }\end{array}$ & A & 10000 & 4449 \\
\hline & & & & & & & & B & 10000 & 4528 \\
\hline \multirow[t]{2}{*}{3} & $17-011$ & Right & $\mathrm{F}$ & 53 & 14.5 & - & $\begin{array}{l}\text { scRNAseq, } \\
\text { cell viability }\end{array}$ & A & 10000 & 4518 \\
\hline & & & & & & & & B & 10000 & 4392 \\
\hline 4 & 16033 & Left & M & 73 & 12 & - & FISH & & & \\
\hline 5 & 16061 & Left & M & 79 & 25 & - & FISH & & & \\
\hline 6 & 16088 & Left & $\mathrm{F}$ & 61 & 22 & - & FISH & & & \\
\hline 7 & 16168 & Left & $\mathrm{M}$ & 74 & 7,13 & - & FISH & & & \\
\hline 8 & $17-080$ & Right & $\mathrm{F}$ & 77 & 4.25 & - & Cell viability & & & \\
\hline 9 & $17-143$ & Left & $\mathrm{M}$ & 66 & 23 & - & Cell viability & & & \\
\hline 10 & $17-153$ & Left & $\mathrm{M}$ & 73 & 13 & - & Cell viability & & & \\
\hline 11 & $17-160$ & Left & $\mathrm{F}$ & 76 & 33.75 & - & Cell viability & & & \\
\hline 12 & $17-167$ & Right & $\mathrm{M}$ & 49 & 16 & - & Cell viability & & & \\
\hline
\end{tabular}


Supplementary table 2: Breakdown of cell number assigned to individual donor libraries. The red highlight the number of cells assigned in the largest clusters, showing similar cell assignment to clusters between 2 replicates.

\begin{tabular}{|c|c|c|c|c|c|}
\hline Clusters & Retina 1 & Retina 2A & Retina 2B & Retina 3A & Retina 3B \\
\hline 0 & 58 & 1927 & 1924 & 103 & 66 \\
\hline 1 & 307 & 33 & 30 & 1419 & 1330 \\
\hline 2 & 3 & 1275 & 1299 & 9 & 9 \\
\hline 3 & 172 & 413 & 425 & 447 & 670 \\
\hline 4 & 74 & 23 & 46 & 999 & 864 \\
\hline 5 & 232 & 59 & 74 & 398 & 384 \\
\hline 6 & 156 & 170 & 139 & 273 & 264 \\
\hline 7 & 737 & 6 & 8 & 42 & 41 \\
\hline 8 & 97 & 98 & 124 & 208 & 190 \\
\hline 9 & 106 & 184 & 189 & 93 & 86 \\
\hline 10 & 33 & 83 & 80 & 180 & 188 \\
\hline 11 & 42 & 83 & 68 & 93 & 77 \\
\hline 12 & 55 & 35 & 35 & 108 & 82 \\
\hline 13 & 18 & 20 & 40 & 58 & 51 \\
\hline 14 & 14 & 7 & 11 & 62 & 71 \\
\hline 15 & 11 & 4 & 12 & 13 & 9 \\
\hline 16 & 5 & 8 & 11 & 9 & 9 \\
\hline 17 & 2 & 21 & 13 & 4 & 1 \\
\hline
\end{tabular}


Supplementary table 3: Membrane-related markers were extracted from GO annotation using the differentially expressed genes in the major human retinal cell types.

Cell types

Rod photoreceptors

Cone photoreceptors

Muller glia

Horizontal cells

Bipolar cells

Amacrinc eclls

Retinal astrocytes

Mieroglia

Retinal ganglion cells

\section{Identified surface markers}

RHO, ROMI, CNGAI, CNGBI, PKHH2, MHGL'

OPN1LW, SLC24A2, DST, TTR

RGR, GPR37, RLBPI, NCAMI, CDI64

CNTNAP2, PTN, NDRG4, TAGLN3

TRPMI, GRMG

EPHB6, SLC5A7, GABRD, KCNJ12, PTPRF, GRN2A

GTPC, SERRLNA5, CD44, SLC4A4, AOP4, ABCC3, NGFR

CD74, IILA-DRA, IILA-DRA1, TYROBR, FCERIG, LAPTMS, CDI4, HLA-DQAI, FXTDS, CTSB, CD37

RTNL, NDRG4, UCHLL, TWHAH 

aCC-BY-NC 4.0 International license.
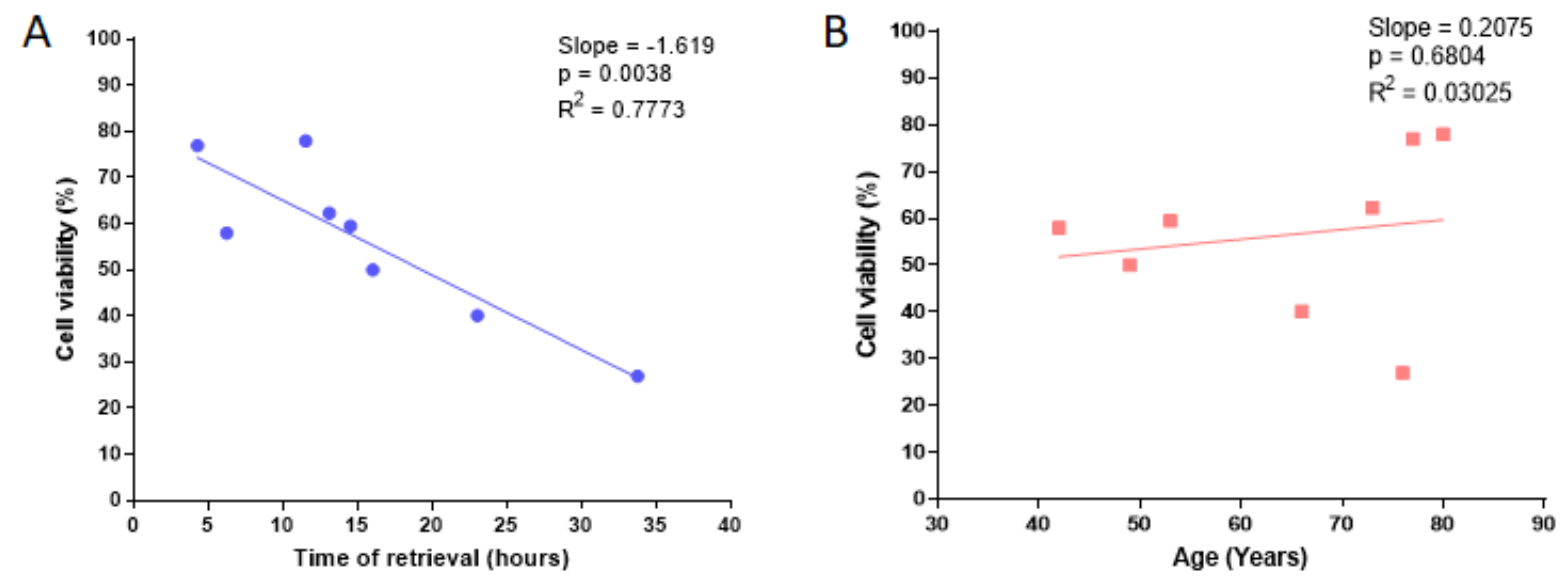

Supplementary figure 1: Correlation of cell viability of post-mortem human neural retina with A) time of tissue retrieval after death, and B) age of donor. 
bioRxiv preprint doi: https://doi.org/10.1101/425223; this version posted Auqust 3 , 2019. The copyright holder for this preprint (which was not certified by peer review) is the author/funder, who has granted bioRxiv a license to display the preprint in perpetuity. It is made available under aCC-BY-NC 4.0 International license.
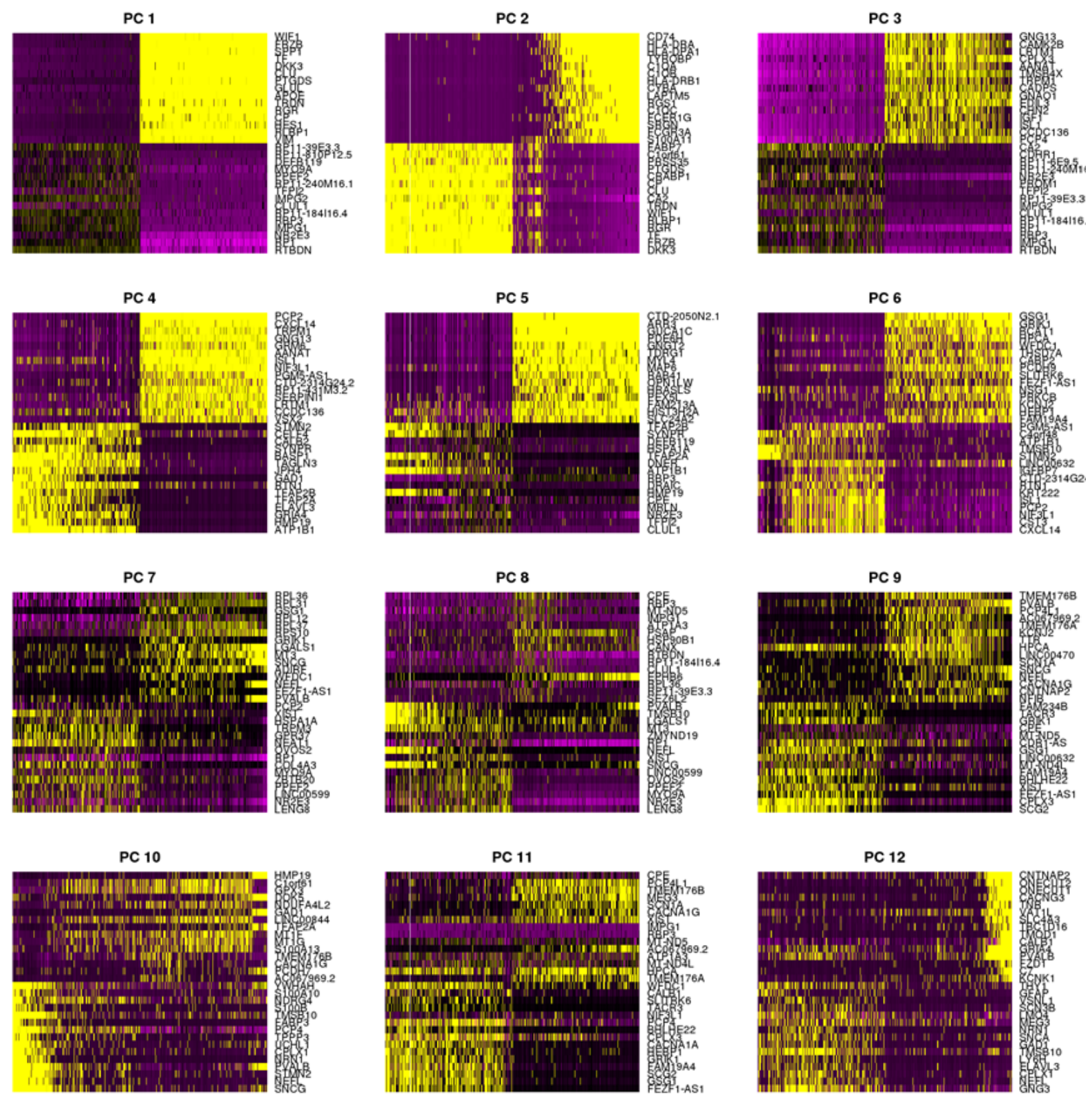

Supplementary figure 2: Heatmaps of the top 12 principal components explaining the primary sources of heterogeneity in the retinal scRNA-seq data. Cells and genes are ordered by PCA score calculated by Seurat. The genes driving the majority of the variance are determined using the top 500 cells. 
A

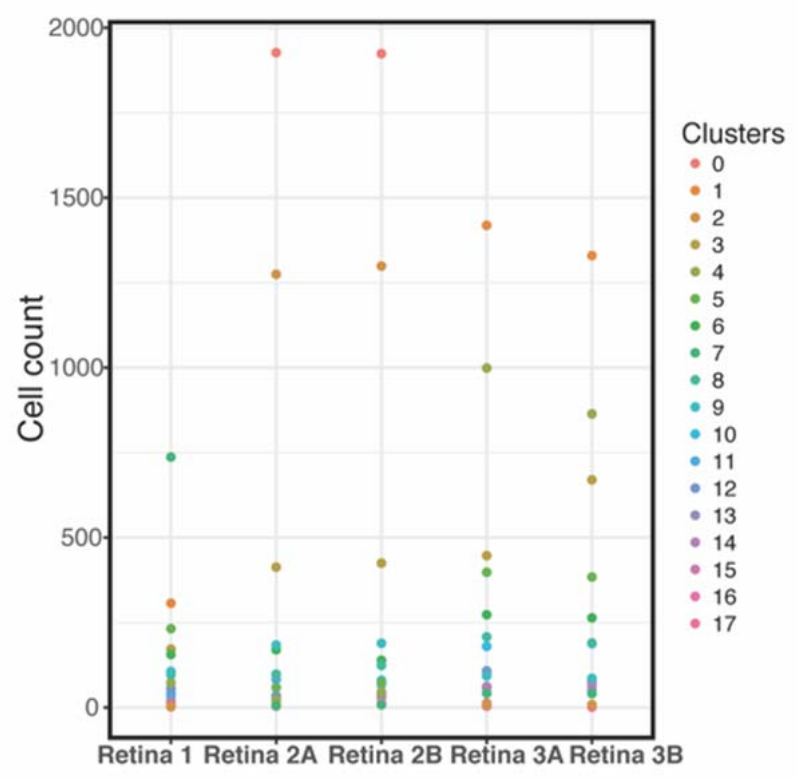

B

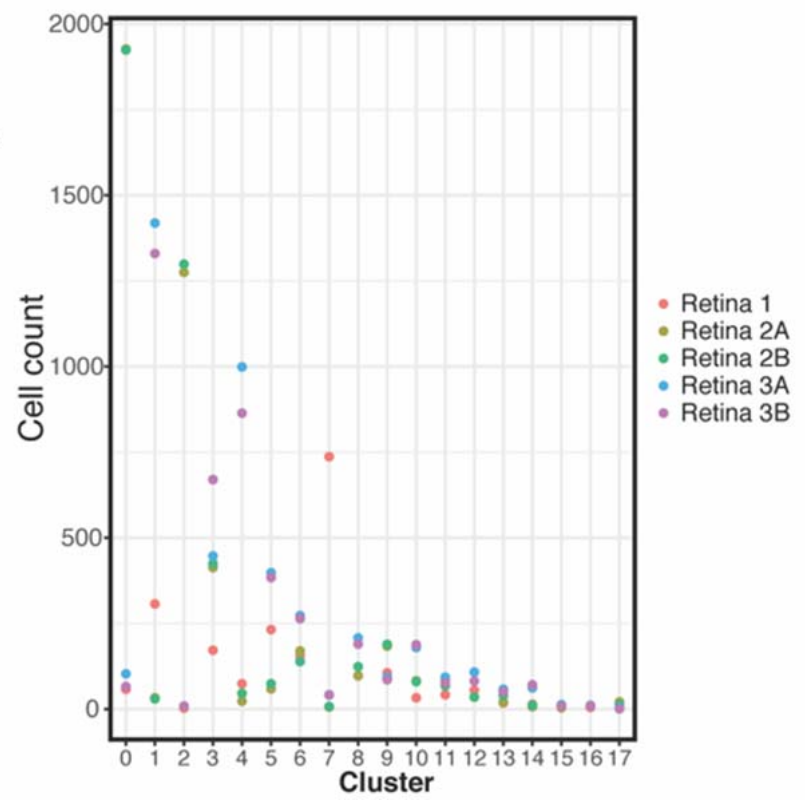

Supplementary figure 3: Distribution of frequency of the 18 clusters $(\mathrm{C} 0-\mathrm{C} 17)$ in individual single cell libraries ordered by A) individual single cell library or B) identified clusters. 


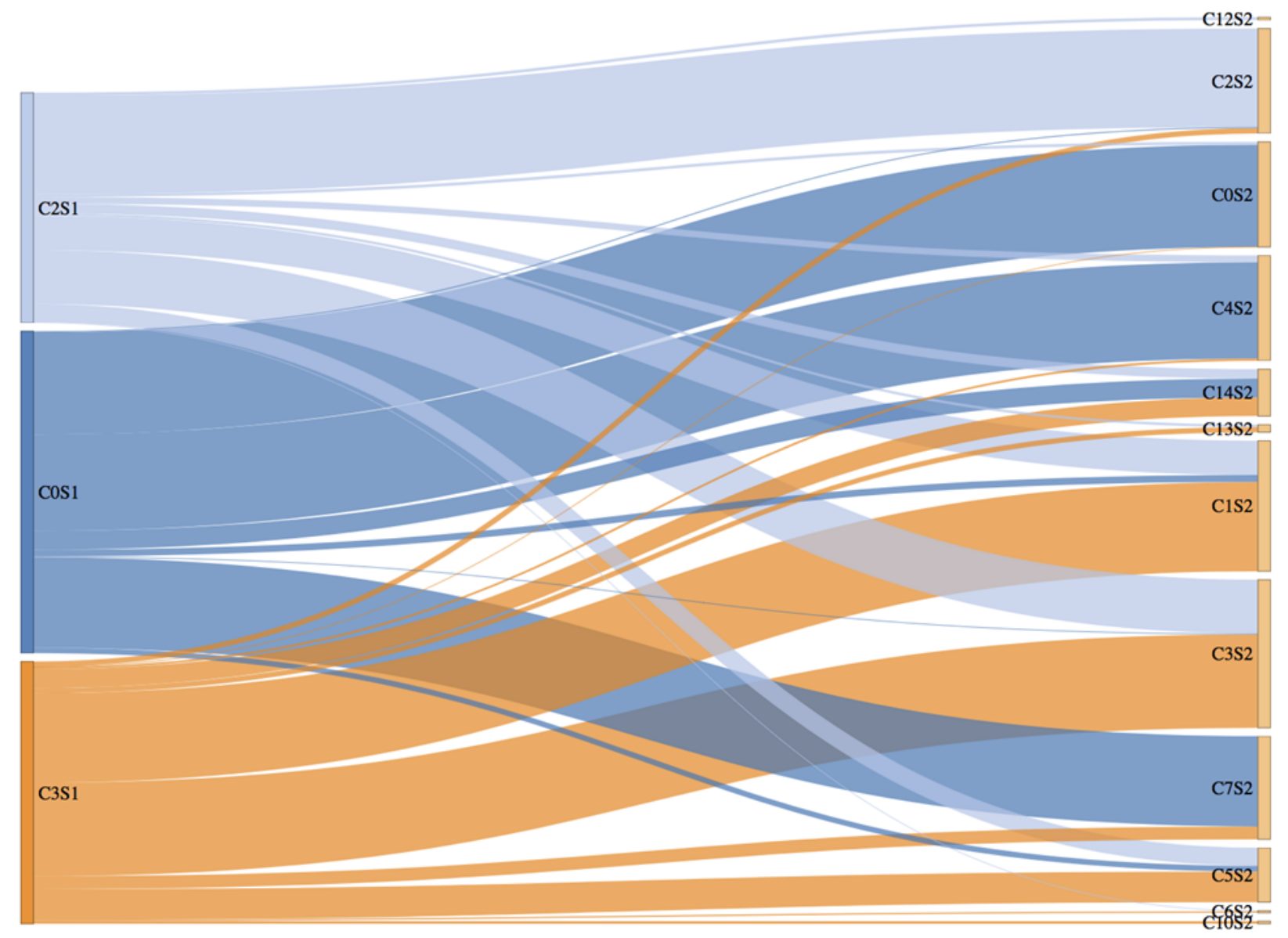

Supplementary figure 4: Prediction of cluster relationship between library technical replicates in Retina 1 and Retina 2. The Sankey plot shows edges connecting clusters, with larger edge indicating higher similarity, ranging from 0 to $100 \%$. The size of the edge was quantitatively estimated by implementing scGPS modelling approach for pairs of clusters, as described in the method section. The three largest clusters in Retina $2 \mathrm{~A}$ were compared with all clusters in Retina 2B. Consistently we see $\mathrm{C} 2$ in Retina $2 \mathrm{~A}$ is most similar to $\mathrm{C} 2$ in Retina $2 \mathrm{~B}$. The same trend is seen for $\mathrm{C} 0$ and $\mathrm{C} 3$. These results demonstrated that the variation between library replicates is minimal in our dataset, and that the clusters determined from the merged dataset were consistent across samples. We also found higher similarities among Rod photoreceptor clusters $(\mathrm{C} 0,2,3$ in Retina 2A with clusters C0, 2, 4, 7 in Retina 2B) than compared with other clusters. 
nTrans
10

$10 \quad 8$
4

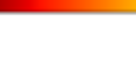

\section{2}

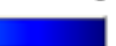

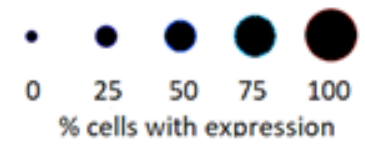

A

Others $\mathrm{C} 14$

Others C5
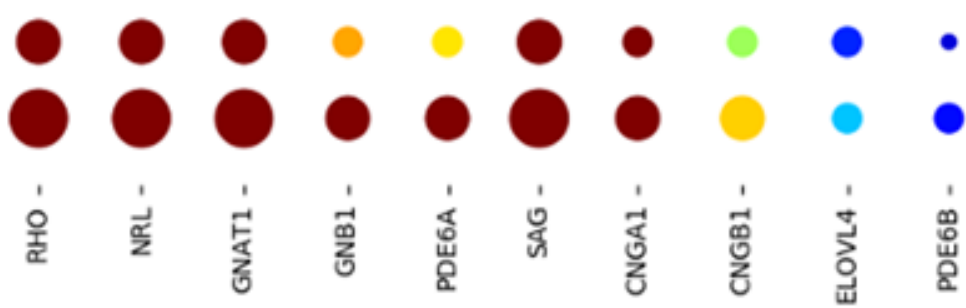

B
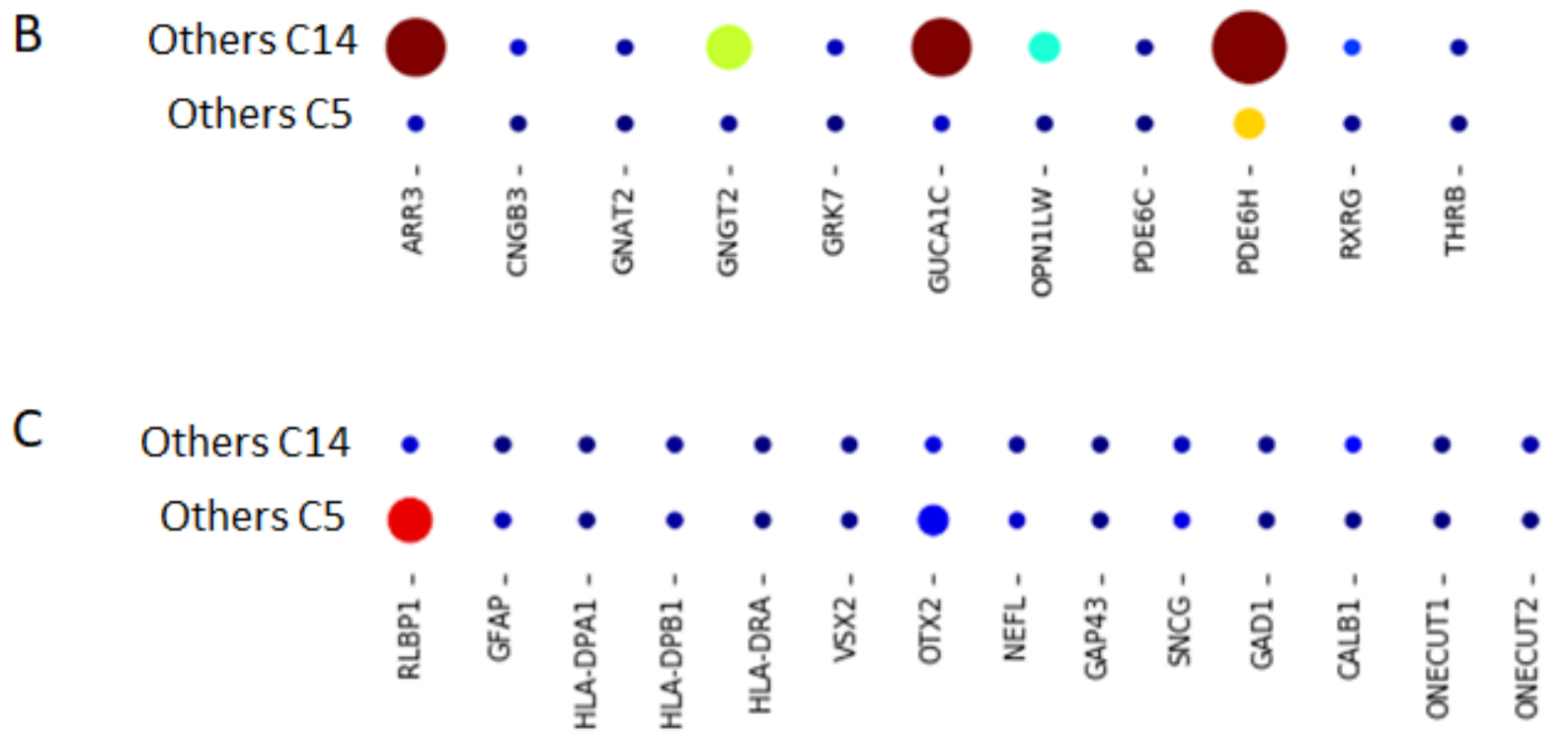

Supplementary figure 5: Feature expression heatmap showing expression patterns of A) rod photoreceptor markers, B) cone photoreceptor markers and C) other major retinal class markers (Müller glia: RLBP1; astrocytes: GFAP; microglia: HLA-DPA1, HLA-DPB1, HLA-DRA; Bipolar cells: VSX2, OTX2; retinal ganglion cells: NEFL, GAP43, SNCG; Amacrine cells: GAD1, CALB1; Horizontal cells: ONECUT1, ONECUT2) in unassigned clusters C14 and C5. The size of each circle depicts the percentage of cells expressing the marker within the cluster. 

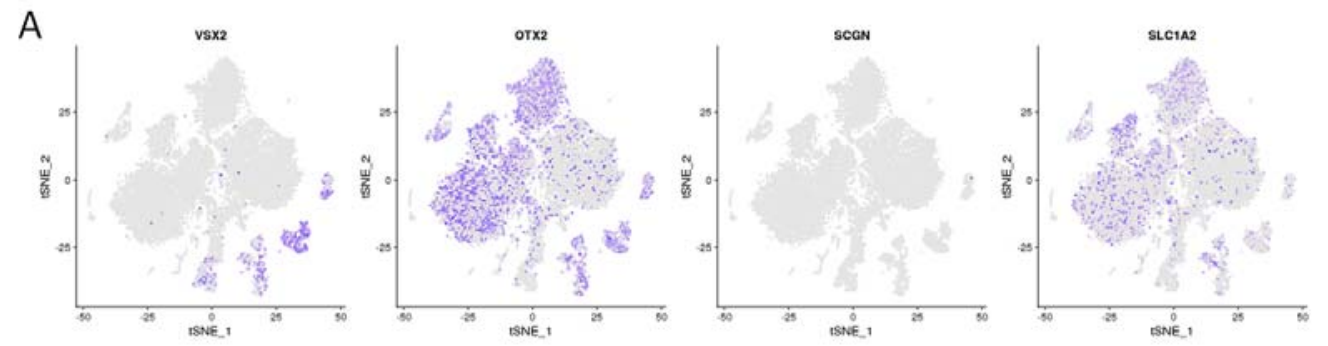

B
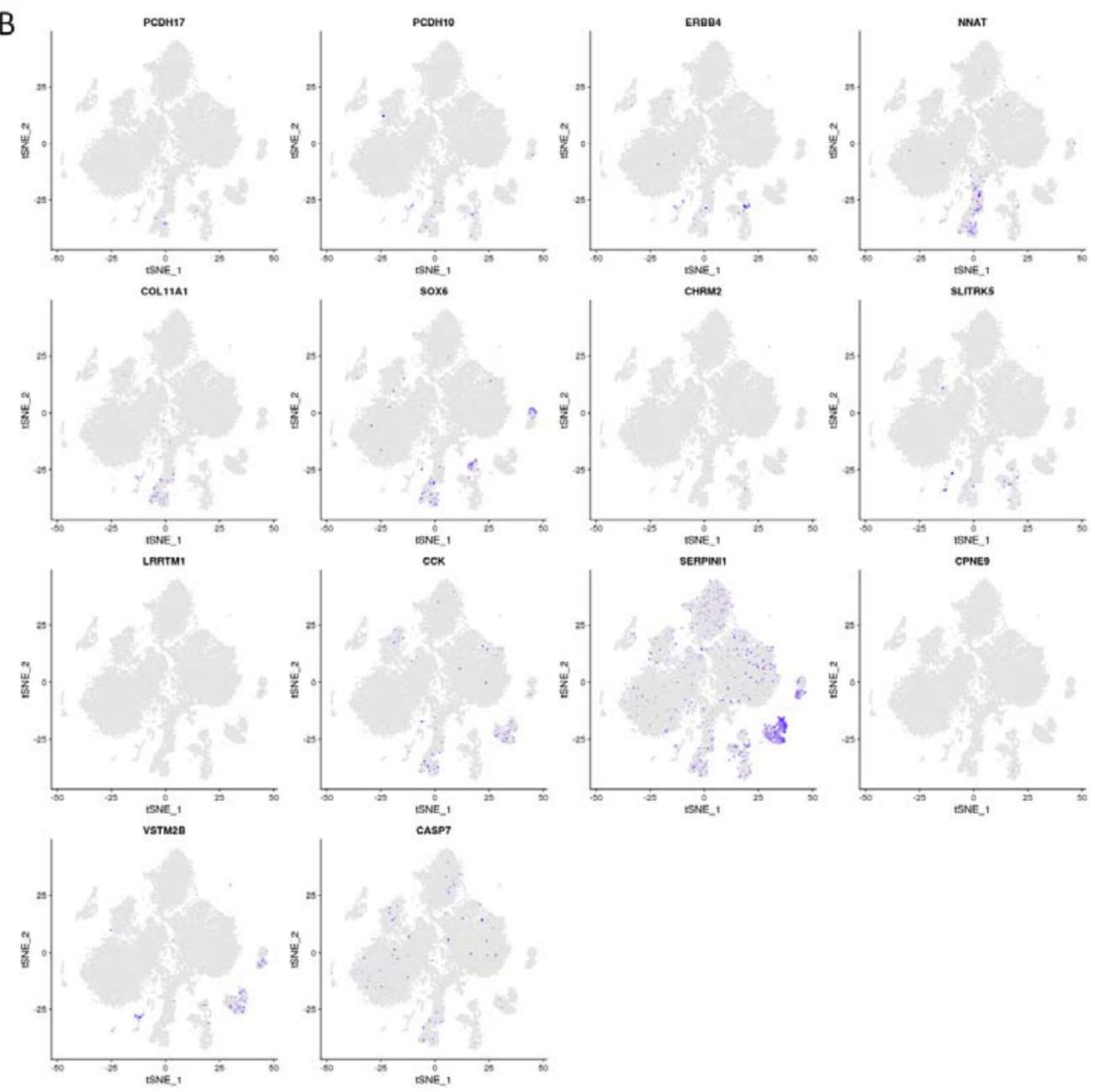

Supplementary figure 6: t-SNE plots showing gene expression in the compiled human neural retina transcriptome atlas (20,009 cells) for A) 4 commonly used bipolar markers and B) 14 new markers for individual bipolar subtypes identified in previous mouse scRNA-seq study (Shekhar et al, 2016). 


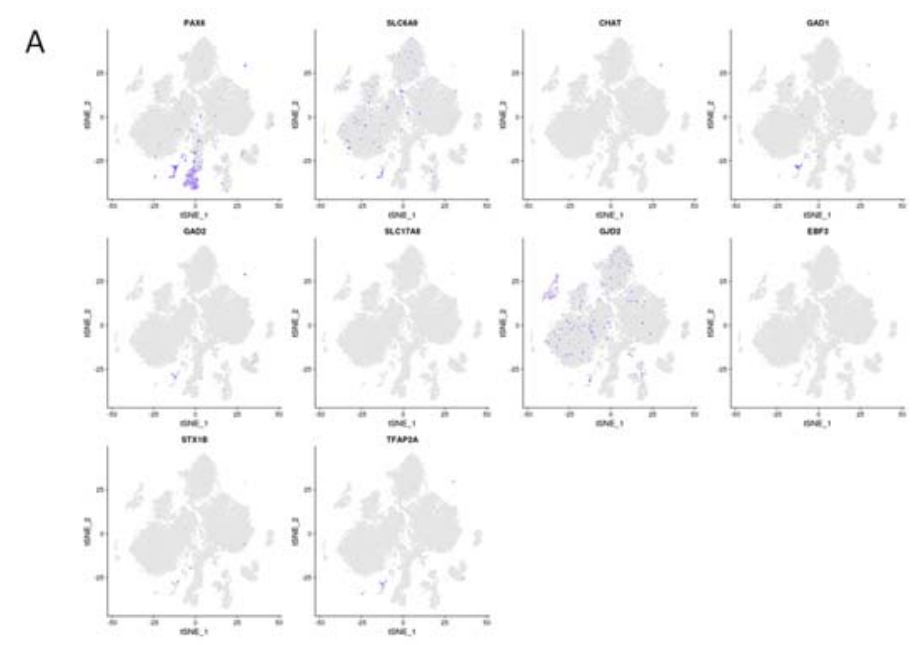

B
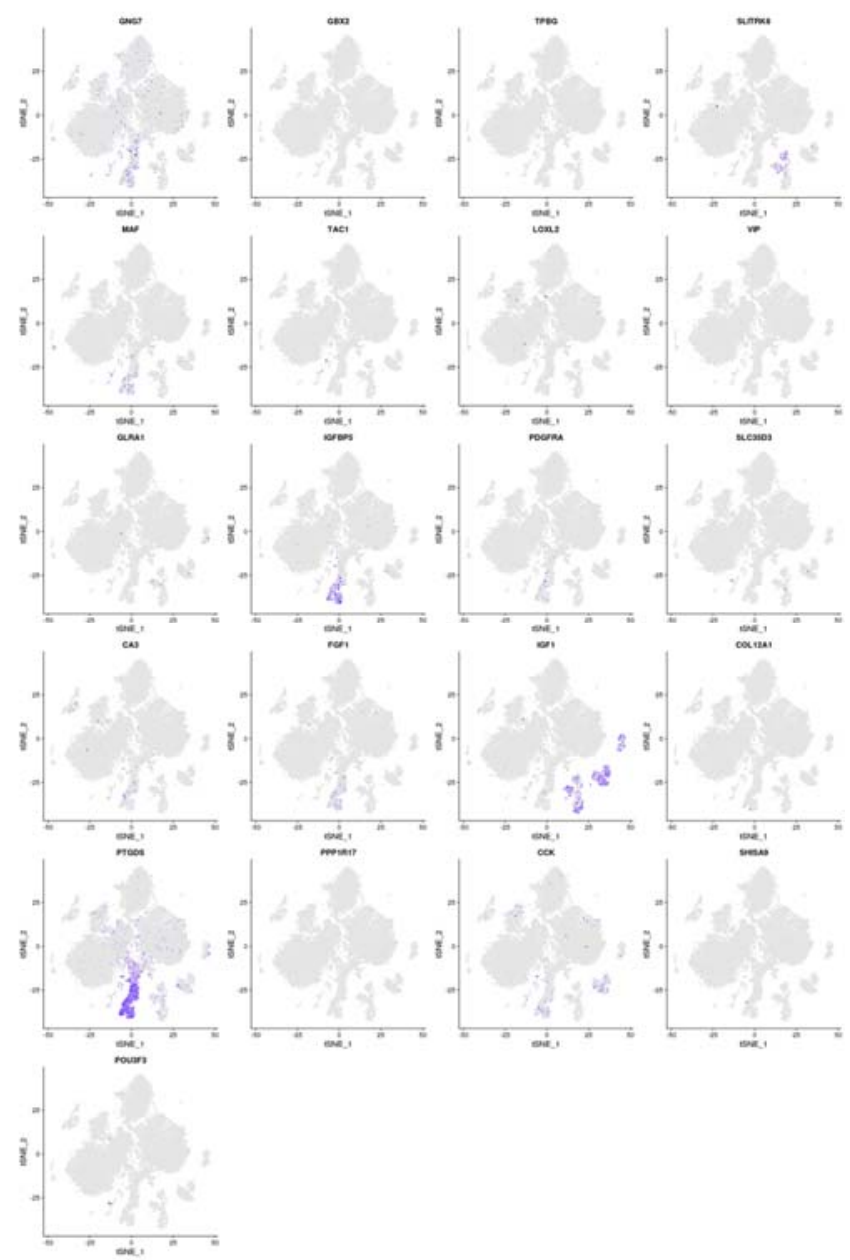

Supplementary figure 7: t-SNE plots showing gene expression in the compiled human retina transcriptome atlas (20,009 cells) for A) 10 commonly used amacrine markers and B) new markers for amacrine subtypes identified in previous mouse scRNA-seq study (Macosko et al, 2015). 


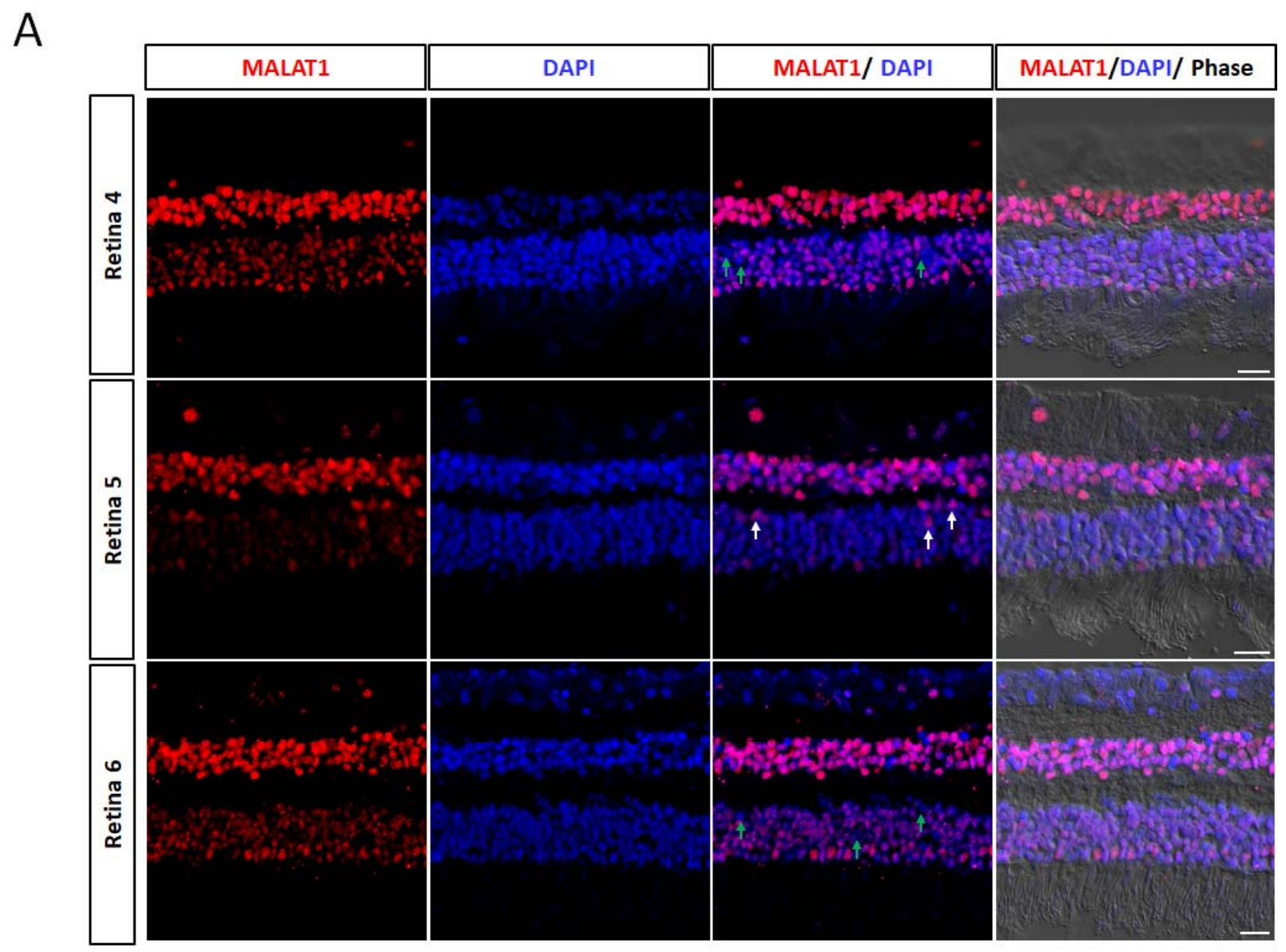

B

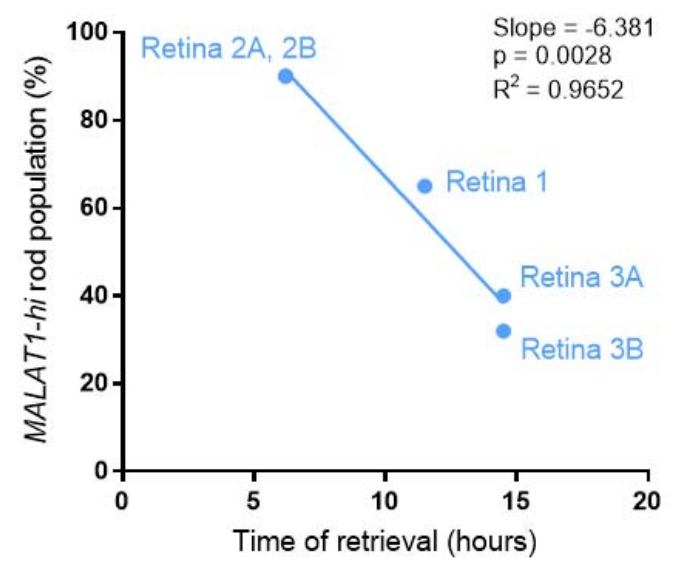

Supplementary figure 8: Fluorescent in situ hybridization showing expression of MALAT1 in three donor retina samples (Retina 4-6). Green arrows highlight rod photoreceptors with low levels of MALAT1 in Retina 4 and 6, white arrows highlight rod photoreceptors with high levels of MALAT1 in Retina 5. Scale bars $=20 \mu \mathrm{m}$. B) Correlation of proportion of MALAT1-hi rod populations with time of retina retrieval after death for Retina 1-3. 

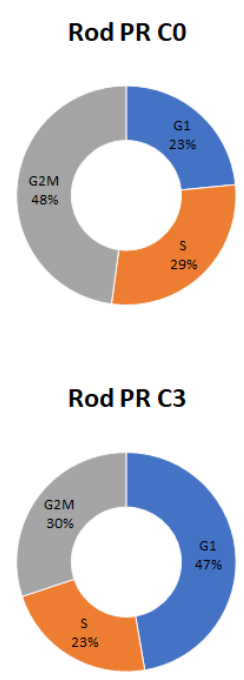

Cone PR C10

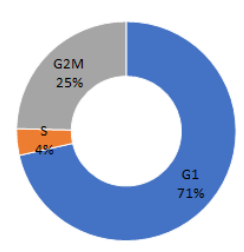

Bipolar cells C6

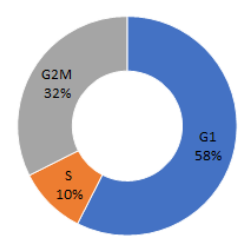

Microglia C13

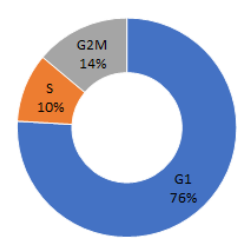

MG C9

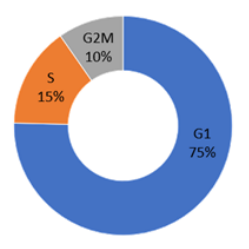

Rod PR C1

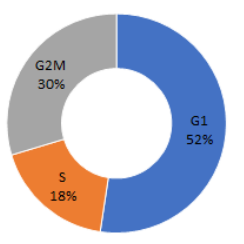

Rod PR C4

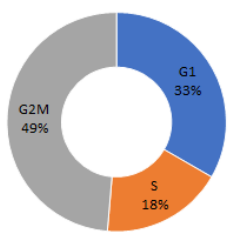

Astrocytes C16

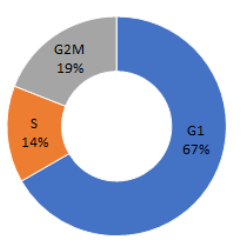

Bipolar cells C8

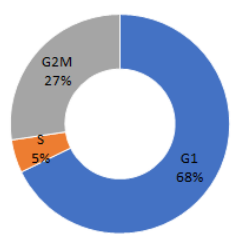

Horizontal cells C15

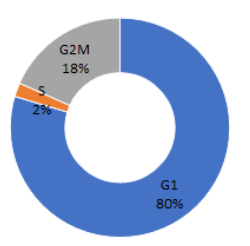

Others C14

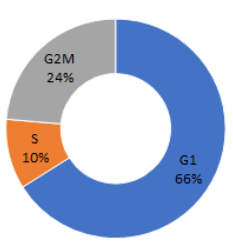

Rod PR C2

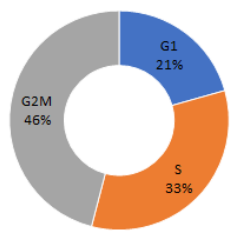

Rod PR C7

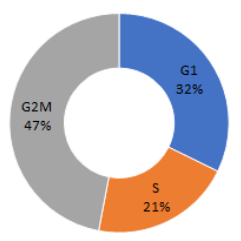

RGC C12

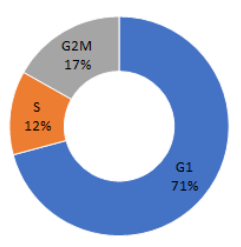

Bipolar cells C11

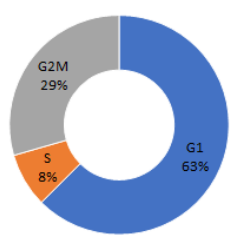

Amacrine cells $\mathrm{C} 17$

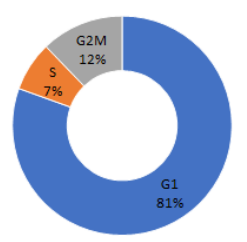

Others C5

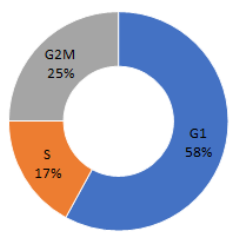

Supplementary figure 9: Cell cycle scores across major retinal cell clusters showing the likelihood for the proportion of cells in $\mathrm{G} 1, \mathrm{~S}$ or $\mathrm{G} 2 / \mathrm{M}$ phases. 


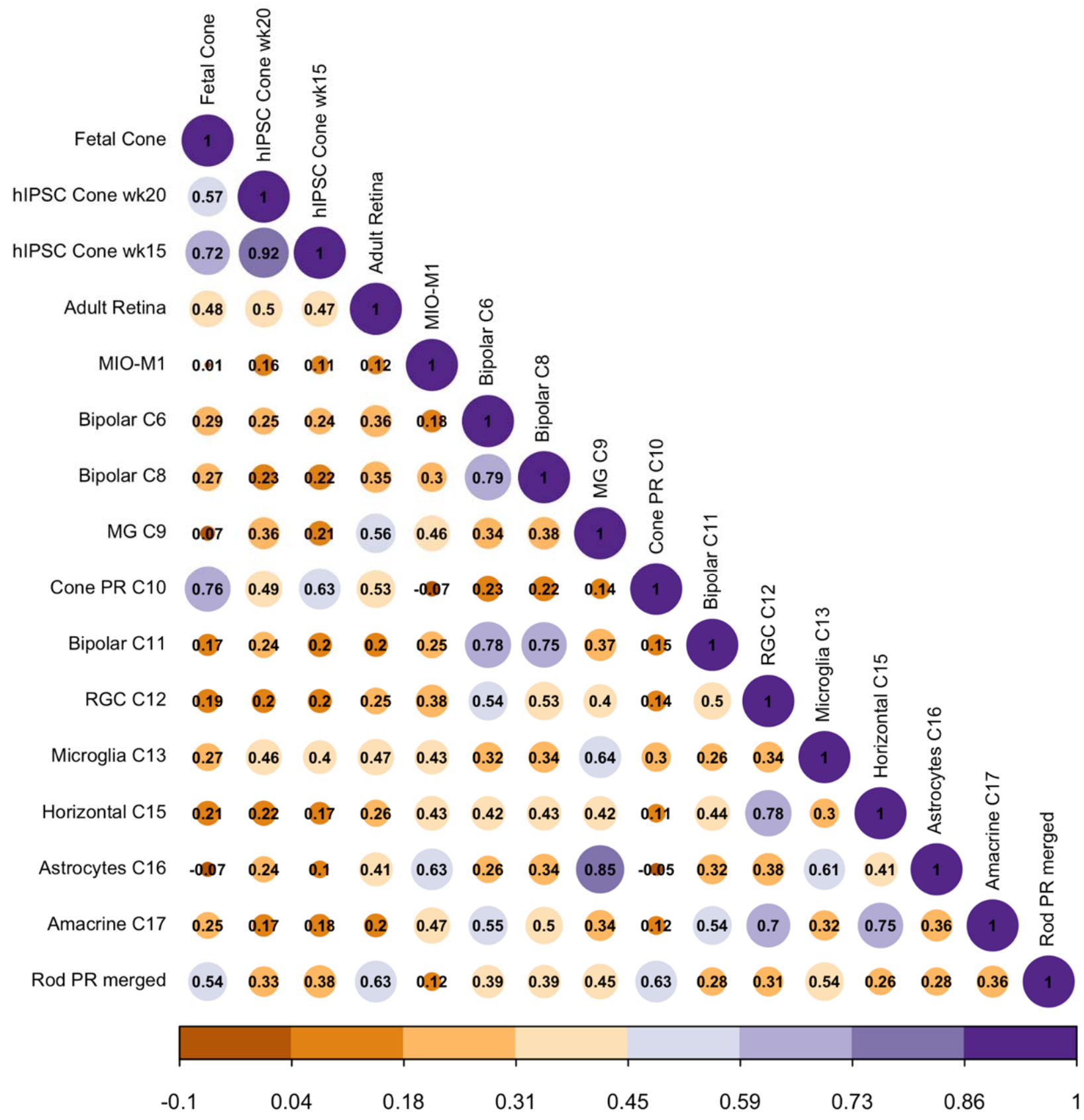

Supplementary figure 10: Correlation matrix to benchmark hiPSC-derived cone photoreceptors (week 15, week 20; (Welby et al, 2017), fetal cone photoreceptors (Welby et al, 2017), adult retina (Phillips et al, 2018) and the human Müller glia cell line MIO-M1 against all retinal cell types identified in this human neural retina atlas. 